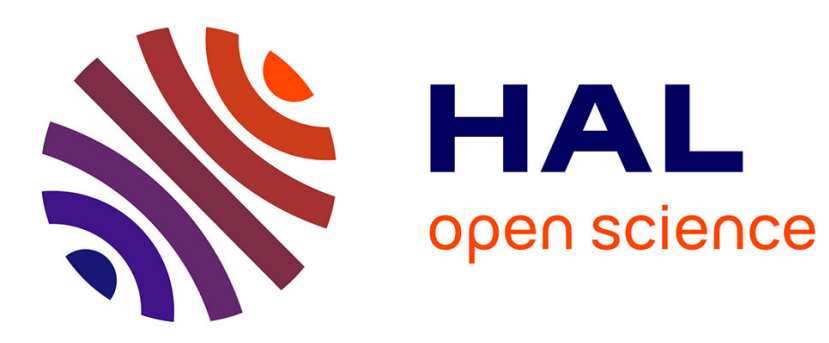

\title{
A fast boundary element based solver for localized inelastic deformations
}

Federico Ciardo, Brice Lecampion, François Fayard, Stéphanie Chaillat

\section{To cite this version:}

Federico Ciardo, Brice Lecampion, François Fayard, Stéphanie Chaillat. A fast boundary element based solver for localized inelastic deformations. International Journal for Numerical Methods in Engineering, 2020, 121 (24), pp.5696 - 5718. 10.1002/nme.6520 . hal-02997409

\section{HAL Id: hal-02997409 \\ https://hal.science/hal-02997409}

Submitted on 26 Nov 2020

HAL is a multi-disciplinary open access archive for the deposit and dissemination of scientific research documents, whether they are published or not. The documents may come from teaching and research institutions in France or abroad, or from public or private research centers.
L'archive ouverte pluridisciplinaire HAL, est destinée au dépôt et à la diffusion de documents scientifiques de niveau recherche, publiés ou non, émanant des établissements d'enseignement et de recherche français ou étrangers, des laboratoires publics ou privés. 


\title{
To cite this document:
}

F.Ciardo, B. Lecampion, F. Fayard, S. Chaillat "A fast boundary element based solver for localized inelastic deformations"

Int. J. Numer. Meth. Engng 121:5696-5718 (2020)

https://doi.org/10.1002/nme.6520

\section{A fast boundary element based solver for localized inelastic deformations}

\author{
F. Ciardo ${ }^{\mathrm{a}}$, B. Lecampion ${ }^{\mathrm{a}, *}$, F. Fayard ${ }^{\mathrm{b}}$, S. Chaillat $^{\mathrm{c}}$ \\ ${ }^{a}$ Geo-Energy Lab - Gaznat chair in Geo-Energy, EPFL, ENAC-IIC-GEL, Lausanne, \\ Switzerland \\ ${ }^{b}$ Inside Loop, France \\ ${ }^{c}$ POEMS (CNRS-ENSTA Paris-INRIA), ENSTA Paris, Institut Polytechnique de Paris,
} France

\begin{abstract}
We present a numerical method for the solution of non-linear geo-mechanical problems involving localized deformation along shear bands and fractures. We leverage the boundary element method to solve for the quasi-static elastic deformation of the medium while rigid-plastic constitutive relations govern the behavior of displacement discontinuity (DD) segments capturing localized deformations. A fully implicit scheme is developed using a hierarchical approximation of the boundary element matrix. Combined with an adequate block pre-conditioner, this allows to tackle large problems via the use of an iterative solver for the solution of the tangent system. Several two-dimensional examples of the initiation and growth of shear-bands and tensile fractures illustrate the capabilities and accuracy of this technique. The method does not exhibit any mesh dependency associated with localization provided that i) the softening length-scale is resolved and ii) the plane of localized deformations is discretized a-priori using DD segments.
\end{abstract}

Keywords: Shear bands, Fractures, Boundary element, hierarchical matrix

1. Introduction

Driven by geomechanical applications such as faulting, shear-banding and 3 fracturing typically occurring in large domains, we develop a computational 4 method for the solution of two dimensional problems exhibiting localized

\footnotetext{
*brice.lecampion@epfl.ch
} 
5

inelastic deformations. We use the boundary element method for the solution of quasi-static elasticity in the medium and accounts for the presence of potential displacement discontinuity (DD) segments where inelastic deformations take place. We use a rigid-plastic like constitutive relation for these DD segments. In particular, we combine a non-associated Mohr-Coulomb frictional behavior with a tensile cut-off, allowing for softening of cohesion, friction and tensile strength. Although the method can be further coupled with fluid flow, we restrict here for clarity to the case where mechanical deformation does not affect flow.

Elasto-plastic problems leading to localized plastic deformations have been extensively investigated using both finite element (FEM) $[1,2,3,4]$ and boundary element (BEM) [5, 6] where in the latter plastic deformations are accounted for via volume integral terms (thus requiring a bulk discretization of the plastic zones [7]). The numerical solutions of this class of non-linear boundary value problems typically exhibit mesh dependencies which are the results of the non-uniqueness associated with the bifurcation of the underlying continuum problem ${ }^{1}[8]$. Several remedies have been proposed to overcome these difficulties: i) introduction of material rate dependence $[9,10]$ which in effect introduce a length-scale, ii) incorporation of a material length-scale in the material constitutive response via gradient based theories [11, 12, 13], non local models [14] or Cosserat continua [15].

In this contribution, we adopt a different approach. Namely, we hypothesise that inelastic deformations can only be localized along displacement discontinuity segments and express the yielding criteria and flow rule only along these segments. This approach shares similarities with cohesive zone modeling in FEM where cohesive traction-separation law between interface element control crack growth $[16,17,18,19]$ and can be traced back to Palmer and Rice [20] for shear band growth. It also resembles the discrete dislocation plasticity method [21]. The use of a boundary element method for the discretization of the DD segments allows to efficiently resolve potential localization phenomena without extensive bulk domain discretization. This is particularly attractive for large domain. Moreover the DD segments are rigid if not at yield thus recovering a solely elastic response in that limit.

In the following, we first present the mathematical formulation of this

\footnotetext{
${ }^{1}$ Strain-softening and non-associated is not necessary in tri-axial setting for localization to occur.
} 
method restricting to a plane strain configuration. The numerical scheme devised as well as the choice of an adequate pre-conditioner for the iterative solution of the resulting tangent system is then discussed in details. We finally illustrate the accuracy and capabilities of this approach on a series of examples involving the initiation and growth of shear-bands and tensile cracks.

\section{Problem formulation}

We consider an homogeneous, isotropic and linear elastic medium under plane-strain condition. The medium is subjected to a generalized system of forces that may cause localized inelastic deformations along a set of predefined segments that translate into displacement discontinuities. A yield criterion controls the occurrence of displacement discontinuities along these segments. If the yield criterion is not satisfied on a particular segment, the displacement discontinuities are zero. Upon yielding, the evolution of displacement discontinuities is governed by a non-associated plastic like flow rule [22]. Incorporating softening, the formalism allows to recover cohesive zone like behavior as well as friction. This enable to capture localized deformations (shear bands, open and sliding fractures). The model is thus akin to a rigid plastic one for the potentially failing segments and elastic for the rest of the solid. This translates into an elasto-plastic response for the whole medium.

\subsection{Elastic medium with displacement discontinuities}

Due to the assumption that inelastic deformations are limited to displacement discontinuity segments, the use of boundary integral equations to solve for the quasi-static elastic balance of momentum is particularly appealing especially for exterior problems. Referring to Figure $1, \Gamma$ denotes the locus of displacement discontinuities, located in a elastic domain $\Omega \in \mathbb{R}^{2}$ with an elastic stiffness tensor $\mathbf{c}_{i j k l}$. We denote the unit normal vector $n_{i}=n_{i}^{-}=-n_{i}^{+}$ where $n_{i}^{+}$and $n_{i}^{-}$are the unit normal vector of the top and bottom surfaces of $\Gamma$ respectively (see Fig. 1). The corresponding shear orthonormal vectors $s$ follow the right-hand side rule. We use the convention of positive displacement discontinuities in opening, positive slip for clock-wise rotation of matter:

$$
d_{i}=u_{i}^{+}-u_{i}^{-}
$$




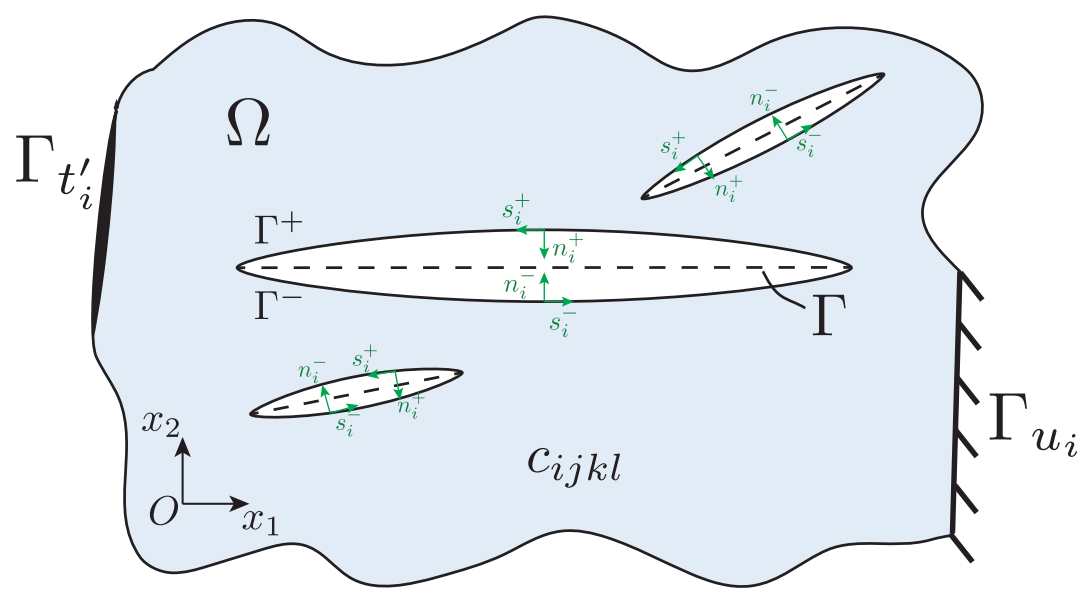

Figure 1: A linearly isotropic elastic medium $\Omega$ containing a set of pre-existing potential fractures and slip planes whose mid-plane are denoted by $\Gamma$. Boundary regions with prescribed displacements or effective traction are denoted respectively as $\Gamma_{u_{i}}$ and $\Gamma_{t_{i}^{\prime}}$.

where $u_{i}$ is the displacement vector. On the other hand, following the convention of geo-mechanics, stresses are taken positive in compression.

The quasi-static elastic equilibrium is written as the following boundary integral equations, relating tractions and displacement discontinuities in the local normal $(n)$ and tangential $(s)$ frame along $\Gamma[23]$ :

$t_{i}(\mathbf{x})-t_{i}^{o}(\mathbf{x})=n_{j}(\mathbf{x}) \int_{\Gamma} c_{i j k l} \frac{\partial S_{a b}^{k}}{\partial \xi_{l}}(\mathbf{x}, \xi) d_{a}(\xi) n_{b}(\xi) \mathrm{d} \xi \quad$ for $\quad \mathbf{x} \in \Gamma, i, j,=n, s$,

where $t_{i}=\sigma_{i j} n_{j}$ is the traction vector, $t_{i}^{o}$ is the initial traction and $S_{a b}^{k}(\mathbf{x}, \xi)$ is the fundamental solution for the stresses at $\xi$ induced by a point force located at $\mathbf{x}$ along the $k$ th direction. $c_{i j k l} \frac{\partial S_{a b}^{k}}{\partial \xi_{l}}(\mathbf{x}, \xi)$ corresponds to the stress induced by a dislocation dipole. We refer to $[23,7,24]$ for more details and expressions for these fundamental elastic solutions. The integral equation (2) is hyper-singular but classical approaches are available in the literature if a collocation [25] or symmetric Galerkin technique [26] is used to drive the discretization.

\subsection{Constitutive relations for displacement discontinuities segments}

We use a Mohr-Coulomb criterion combined with a tensile cut-off as the yielding function for localized failure on segments, allowing for softening (see Figure 2). Accounting for the presence of fluid (of pressure $p$ ), we combine 


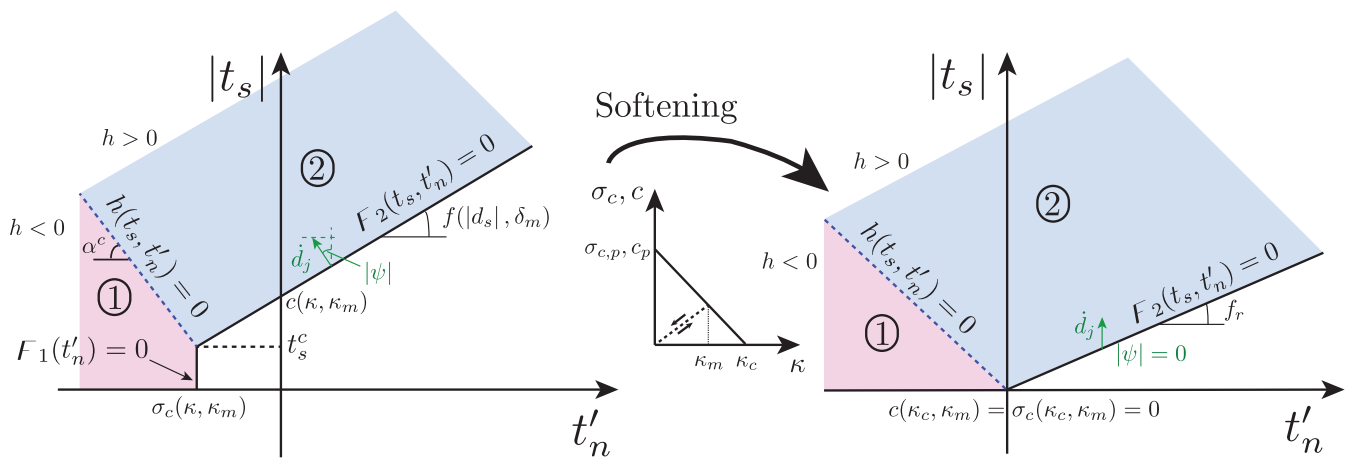

Figure 2: Composite yielding surface for displacement discontinuity segments combining a Mohr-Coulomb (region 2) with a tensile cut-off (region 1) - left panel. Softening of tensile strength, cohesion as well as friction is possible ultimately resulting in a purely frictional behavior at complete softening - right panel. A non-associated flow rule for the frictional response limit plastic dilatancy and result in critical state flow at complete softening (right).

two yield functions expressed in terms of the local components of effective traction vector $t_{n}^{\prime}=t_{n}-p, t_{s}^{\prime}=t_{s}$ :

$$
\begin{aligned}
& \digamma_{1}\left(t_{n}^{\prime}\right)=-\sigma_{c}\left(\kappa, \kappa_{m}\right)-t_{n}^{\prime} \leq 0, \\
& \digamma_{2}\left(t_{s}, t_{n}^{\prime}\right)=\left|t_{s}\right|-c\left(\kappa, \kappa_{m}\right)-f\left(\left|d_{s}\right|, \delta_{m}\right) t_{m}^{\prime} \leq 0,
\end{aligned}
$$

where $f\left(\left|d_{s}\right|, \delta_{m}\right)$ is the friction coefficient function of absolute value of shear slip $d_{s}$ and the maximum slip obtained during the loading history $\delta_{m}$. Similarly, $\sigma_{c}\left(\kappa, \kappa_{m}\right)$ and $c\left(\kappa, \kappa_{m}\right)$ are the tensile strength and cohesion respectively, both function of a softening variable $\kappa=\sqrt{\xi^{2} d_{s}^{2}+d_{n}^{2}}$, where $\xi>0$ is a phenomenological parameter accounting for the relative intensity of shear and normal displacement on softening. $\kappa_{m}$ corresponds to the maximum value of $\kappa$ obtained during the loading history.

In order to define uniquely which yield function the effective traction vector must satisfy when both criteria are violated simultaneously (when $\digamma_{1}\left(t_{n}^{\prime}\right)>0$ and $\digamma_{2}\left(t_{s}, t_{n}^{\prime}\right)>0$ ), we use a function $h\left(t_{s}, t_{n}^{\prime}\right)$ similar to the one proposed in [27]

$$
h\left(t_{s}, t_{n}^{\prime}\right)=\left|t_{s}\right|-t_{s}^{c}-\alpha^{c}\left(\sigma_{c}\left(\kappa, \kappa_{m}\right)+t_{n}^{\prime}\right),
$$

where $t_{s}^{c}$ and $\alpha^{c}$ are two scalars function of the current friction, cohesion and tensile strength defined as 


$$
\begin{aligned}
t_{s}^{c} & =c\left(\kappa, \kappa_{m}\right)-f\left(\left|d_{s}\right|, \delta_{m}\right) \sigma_{c}\left(\kappa, \kappa_{m}\right) \\
\alpha^{c} & =\sqrt{1+f\left(\left|d_{s}\right|, \delta_{m}\right)^{2}}-f\left(\left|d_{s}\right|, \delta_{m}\right)
\end{aligned}
$$

The yielding functions $\digamma_{1}\left(t_{n}^{\prime}\right)$ and $\digamma_{2}\left(t_{s}, t_{n}^{\prime}\right)$ represent inequality constraints for the traction applied on $\Gamma$. Combined with the function $h\left(t_{s}, t_{n}^{\prime}\right)$, they allow to split uniquely the effective traction space into admissible and inadmissible regions (see Figure 2): specifically, $\digamma_{2}\left(t_{s}, t_{n}^{\prime}\right)$ for $h\left(t_{s}, t_{n}^{\prime}\right) \geq 0$ (shear failure) and $\digamma_{1}\left(t_{n}^{\prime}\right)$ for $h\left(t_{s}, t_{n}^{\prime}\right)<0$. In the following, we describe the relations that the local tractions must satisfy on a given displacement discontinuity segment $\Gamma$ for the different inadmissible regions 1 and 2 of Figure 2 corresponding to tensile or shear failure respectively.

\subsubsection{Shear failure}

Shear failure is captured via a non-associated flow rule to better reproduce shear-induced dilatancy (with a dilatant angle typically lower than friction angle). The yield criteria constraint and corresponding evolution of the displacement discontinuity rates are thus similar to frictional contact with cohesion:

$$
\begin{array}{ll}
\digamma_{2}\left(t_{s}, t_{n}^{\prime}\right)<0, & \dot{d}_{s}=0, \quad \dot{d}_{n}=0 \\
\digamma_{2}\left(t_{s}, t_{n}^{\prime}\right)=0, & \dot{d}_{s}=\left|\dot{d}_{s}\right| \operatorname{sign}\left(t_{s}\right), \quad \dot{d}_{n}=\left|\dot{d}_{s}\right| \tan \psi\left(\left|d_{s}\right|, \delta_{m} \mid\right)
\end{array}
$$

During shear failure, the evolution of cohesion $c$ and friction coefficient $f$ with non-linear deformations governs the traction separation along $\Gamma$. We assume that the cohesion $c$ degrades linearly with softening variable $\kappa$ in a similar way than the tensile strength $\sigma_{c}$ (see the following sub-section) keeping the ratio $c / \sigma_{c}$ constant. The friction coefficient $f$ is supposed to weaken linearly with the absolute value of slip $\left|d_{s}\right|$, from a peak value $f_{p}$ to a residual value $f_{r}$ for slip larger than a critical slipping distance $\delta_{c}$ [20]:

$$
f\left(\left|d_{s}\right|, \delta_{m}\right)= \begin{cases}f_{p}-\frac{f_{p}-f_{r}}{\delta_{c}}\left|d_{s}\right| & \left|d_{s}\right|<\delta_{c} \quad \& \quad\left|d_{s}\right|=\delta_{m} \\ f_{p}-\frac{f_{p}-f_{r}}{\delta_{c}} \delta_{m} & \left|d_{s}\right|<\delta_{c} \& \quad\left|d_{s}\right|<\delta_{m} \\ f_{r} & \left|d_{s}\right|>\delta_{c}\end{cases}
$$

Similarly, we assume that the dilatancy angle $\tan \psi$ softens linearly with cumulative slip $\left|d_{s}\right|$, from a peak value $\tan \psi_{p}$ down to zero above a critical slip distance $\delta_{c}$ at which a critical state is reached [28]. Like for the friction 
coefficient, the dilatancy angle does not evolve along the unloading/reloading branch. Although one can expect a drop of dilation angle during reverse deformation (see Stupkiewicz and Mróz [29] for discussion), we stick to that assumption for sake of simplicity in the following.

\subsubsection{Tensile failure}

Tensile failure on $\Gamma$ (inadmissible region 1 of Figure 2) is directly controlled by the value of the effective normal traction. The relations for the evolution of the displacement discontinuities are here given by:

$$
\begin{array}{llrl}
\digamma_{1}\left(t_{n}^{\prime}\right)<0, & \dot{d}_{n}=0, & \dot{d}_{s}=0 \\
\digamma_{1}\left(t_{n}^{\prime}\right)=0, & \left|\dot{d}_{n}\right|>0, & \dot{d}_{s}=0
\end{array}
$$

with the complementary condition $\digamma_{1}\left(t_{n}^{\prime}\right)\left|\dot{d}_{n}\right|=0$. The sign of $\dot{d}_{n}$ depends on the loading / unloading sequence and results from the application of the constraint $\digamma_{1}\left(t_{n}^{\prime}\right)=0$ in the solution of the balance of momentum.

The evolution of the critical tensile strength $\sigma_{c}$ with softening variable $\kappa$ governs the relation between tractions and displacement discontinuities along $\Gamma$ in a similar way than in cohesive zone models of fracture $[17,30,31]$. In the following, we assume that $\sigma_{c}$ softens linearly with $\kappa$, from a peak value $\sigma_{c, p}$ to zero when $\kappa$ is larger than a critical value $\kappa_{c}$. We also account for a reversible linear unloading/re-loading branch when the softening variable $\kappa$ is lower than its maximum value reached during the loading history $\kappa_{m}$ (see Figure 2). This can be summarized as

$$
\frac{\sigma_{c}\left(\kappa, \kappa_{m}\right)}{\sigma_{c, p}}= \begin{cases}1-\kappa / \kappa_{c} & \kappa<\kappa_{c} \& \kappa=\kappa_{m} \\ \left(1-\kappa_{m} / \kappa_{c}\right) \kappa / \kappa_{m} & \kappa<\kappa_{c} \& \kappa<\kappa_{m} \\ 0 & \kappa>\kappa_{c}\end{cases}
$$

At complete softening, both the tensile strength $\sigma_{c}$ and the cohesion $c$ are zero resulting in a purely frictional Mohr-Coulomb criterion (see Figure 2 right). As a result, if $\digamma_{1}\left(t_{n}^{\prime}\right)=0$ at complete softening (i.e. $t_{n}^{\prime}=0$ ), one must also enforce $t_{s}=0$ (i.e. $\digamma_{2}\left(t_{s}, t_{n}^{\prime}\right)=0$ ) and as a result $\left|\dot{d}_{n}\right|>0,\left|\dot{d}_{s}\right|>0$.

Non inter-penetrability constraint at closure

When the tensile mode I failure is active, the sign of the normal displacement discontinuity rate is the result of the elastic balance of momentum of the whole medium, boundary conditions and the associated interactions between 
failed segments. Upon unloading, crack closure is possible. Of course, the internal crack surfaces can not interpenetrate. Accounting for the irreversible dilation $\bar{w}_{d}=\int_{0} t \tan \psi(\kappa) \dot{d}_{s} \mathrm{~d} t$ accumulated during the loading history, we generalize the non inter-penetrability condition to

$$
\left(d_{n}-w_{d}\right) \geq 0 \quad \digamma_{1}\left(t_{n}^{\prime}\right) \leq 0 \quad\left(d_{n}-w_{d}\right) \digamma_{1}\left(t_{n}^{\prime}\right)=0
$$

\subsection{Initial and boundary conditions}

We assume that the elastic medium is initially in static equilibrium under a initial stress field $\sigma_{i j}^{o}$ resulting in traction $t_{i}^{o}$ on $\Gamma$. We assume that the initial state is such that the yielding criterion is not violated in any potential displacement discontinuity segments. Localized inelastic deformations therefore occurs as a result of either external loading (via an history of applied loads or displacements) or via internal pore fluid pressurization $p$ which modifies the effective traction on the potential failure segments. We assume here the pore-pressure history known and uncoupled to mechanical deformation. Such time-dependent boundary conditions can be summarized as (in the local frame $i=s$, of the boundary):

$$
t_{i}^{\prime}(\mathbf{x}, t)=t_{i}^{g}(\mathbf{x}, t)-p(\mathbf{x}, t) \quad \text { on } \quad \Gamma_{t_{i}^{\prime}}
$$

$$
u_{i}(\mathbf{x}, t)=u_{i}^{g}(\mathbf{x}, t) \quad \text { on } \quad \Gamma_{u_{i}}
$$

with the usual conditions $\Gamma=\Gamma_{u_{i}} \cup \Gamma_{t_{i}^{\prime}}$, and $\Gamma_{u_{i}} \cap \Gamma_{t_{i}^{\prime}}=\emptyset \cdot t_{i}^{g}(\mathbf{x}, t), u_{i}^{g}(\mathbf{x}, t)$ and $p$ denotes given applied traction vector, displacement components and fluid pore pressure respectively. Note that in the absence of fluid, the pressure $p$ is null and $t_{i}^{\prime}$ reduces to $t_{i}$.

\section{Numerical scheme}

\subsection{Boundary element method for elasto-static using a hierarchical matrix approximation}

We use the displacement discontinuity method [25] to discretize the elasticity equations (2). Upon discretization of $\Gamma$ (union of all possible failing segments) into $n_{\text {segm }}$ straight segments such that

$$
\Gamma \approx \bigcup_{s=1}^{n_{\text {segm }}} \Gamma_{s}
$$


We assume that displacement discontinuities $d_{i}$ vary linearly within an element but discontinuously between adjacent elements (piece-wise linear element). This assumption sets a weaker requirement at each intersecting mesh node $n_{\text {node }}=n_{\text {segm }}+1$ (i.e. no continuity of displacement discontinuities), which notably allows to treat configurations of fractures intersection more easily. For $n_{\text {segm }}$ straight finite segments, we thus have $n=4 n_{\text {segm }}$ nodal displacement discontinuities unknowns. By introducing this discretization into the boundary integral elasticity equations (2), using a collocation method, one finally obtain a $4 n_{\text {segm }} \times 4 n_{\text {segm }}$ linear system of equations

$$
\mathbf{t}=\mathbf{t}^{o}+\mathbf{E d}
$$

where $\mathbf{t}$ and $\mathbf{t}^{o}$ are respectively the current and far-field traction vectors, $\mathbf{E}$ is the fully populated elastic influence matrix and $\mathbf{d}$ is the vector of nodal displacement discontinuities. Because of the singular nature of equation (2), collocation is performed at points located inside the displacement discontinuity element - see [32] for discussion on their optimal location within the reference straight element.

Due to the non-locality of the elasticity kernel, the elasticity matrix $\mathbf{E}$ is fully populated although diagonal dominant. The memory requirement to store such a square matrix thus scales as $\mathcal{O}\left(n^{2}\right)$, setting a strict constraint for current available laptops with 64-bit processors. Furthermore, the computational complexity to solve the system of equations (13) with an iterative method is $\mathcal{O}\left(k \cdot n^{2}\right)$ (where $k$ is the number of iterations to reach convergence in the iterative solver, with possibly $k \ll n$ if the system is well-conditioned). In order to overcome these limits, we use a hierarchical matrix ( $\mathcal{H}$-matrix) representation of the BEM matrix combined with adaptive cross approximation to perform low-rank approximations [33]. This purely algebraic acceleration technique makes use of the spatial decay of the elastic kernel to approximate its far-field contributions via a data-sparse representation (low rank approximation). This allows to reduce memory requirements and, at the same time, speed up algebraic operations [34, 35]. First, a geometrical binary tree $\mathcal{T}_{\mathcal{I}}$ associated with the location of the collocation points is built. Its maximum depth is governed by a scalar parameter $n_{\text {leaf }}$ that defines the minimum cardinality of each cluster. Upon recursive evaluation of the following admissibility condition

$$
\operatorname{Adm}(p, q)=\operatorname{true} \Longleftrightarrow \min \{\operatorname{diam}(p), \operatorname{diam}(q)\} \leq \eta \cdot \operatorname{dist}(p, q),
$$


to all the pair-nodes composing the block cluster tree $\mathcal{T}_{\mathcal{I}}$, a partitioning of the elastic matrix into admissible (far-field) and inadmissible (near-field) blocks is obtained. The diameter of a generic cluster $p \in \mathcal{T}_{\mathcal{I}}$ is defined as

$$
\operatorname{diam}(p):=\max _{i, j \in p}\left\|x_{i}-x_{j}\right\|
$$

and the distance between two clusters $p, q \in \mathcal{T}_{\mathcal{I}}$ is

$$
\operatorname{dist}(p, q):=\min _{i \in p, j \in q}\left\|x_{i}-x_{j}\right\| \text {. }
$$

The admissible blocks are approximated via low-rank matrices obtained with an adaptive cross approximation technique (see [34, 35, 36] for full details). Non-admissible blocks are stored and treated as dense matrices (full rank representation). It can be proved that by replacing the full elasticity matrix $\mathbf{E}$ with its hierarchical approximation $\mathbf{E}_{\mathcal{H}}$, the generic computational complexity reduces to $[35] \mathcal{O}(n \times \log (n))$ for storage requirements and matrix-vector multiplications.

The construction of the $\mathcal{H}$-matrix representation of the initial matrix depends on 3 parameters: i) $\eta \geq 0$ governs the severity of the clustering (i.e. large value of $\eta$ promote a more aggressive block partitioning, while $\eta=0$ results in no partitioning, i.e. $\mathbf{E}_{\mathcal{H}}=\mathbf{E}$ ), ii) $n_{\text {leaf }}>0$ defines the maximum depth of the block cluster tree $\mathcal{T}_{\mathcal{I}}$ and iii) $\epsilon_{A C A}$ governs the accuracy of the low-rank approximation obtained via an adaptive cross approximation (see [34] for details for scalar problems and [36] for vector problems). The gain in memory storage with respect to the initial dense matrix is quantified by the memory compression ratio $c_{r}$ given by

$$
c_{r}\left(E_{\mathcal{H}}\right)=\frac{1}{n^{2}}\left(\sum_{(p, q) \in \mathrm{Adm} .} \operatorname{rank} \cdot(|p|+|q|)+\sum_{(p, q) \in \text { Non-Adm. }}|p| \cdot|q|\right)
$$

while the accuracy of $\mathbf{E}_{\mathcal{H}}$ is function of $\eta, n_{\text {leaf }}$ and $\epsilon_{A C A}$. In the remaining, we consider only a hierarchical approximation $\mathbf{E}_{\mathcal{H}}$ of the elasticity matrix.

\subsection{An implicit time-stepping scheme}

For a given load / pore pressure history, the solution of the problem consists in the solution of the discretized elasto-static balance of momentum in combination with the set of inequalities constraints introduced in section 2.2. Besides the inequalities, softening reinforces the non-linearity of the 
problem. We use an implicit time-stepping scheme to obtain the solution at $t^{n+1}=t^{n}+\Delta t$ from a known solution at $t^{n}$. We solve for both the evolution of the displacement discontinuities as well as the corresponding tractions over the whole discretized mesh $\Gamma$. We use the notation $X^{n+1}=X^{n}+\Delta X$ to represent a generic time and space dependent variable $X(x, t)$ at time $t^{n+1}$. Over a time-step, the algorithm consists of two nested loops. The outer loop tracks the set of elements satisfying the yielding constraints and non interpenetrability condition. The inner loop - for a given trial set of constraints - solves for the balance of momentum, and enforces the different equality constraints. Softening renders such an inner loop non-linear and we thus use a fixed-point scheme for its solution.

\subsubsection{Outer yielding loop}

The outer iterative loop is used to converge on the different inequalities constraints (yielding and non inter-penetrability conditions) for all the elements within the mesh. At each iteration, the algorithm must identify the set of elements $\mathcal{S}_{a, 1}$ active in tensile failure (satisfying eq. (3a)), the set of elements $\mathcal{S}_{a, 2}$ active in shear failure (satisfying eq. (3b)), and the set of elements $\mathcal{S}_{\text {interp. }}$ violating the inter-penetrability constraint eq. (9). The set of inactive elements (neither yield or interpenetrating) $\mathcal{S}_{\text {inact. }}$ is just the complement

$$
\mathcal{S}_{\text {inact. }} \notin\left\{\mathcal{S}_{a, I} \cup \mathcal{S}_{a, I I} \cup \mathcal{S}_{\text {interp. }}\right\}
$$

such that the union of all these sets equals the total number of elements in the mesh. A priori, these sets are unknown. Over a load/time step, during this iterative procedure, an element can thus switch from being inactive (not violating either the yield or contact conditions) to being active - where then the yield constraints are enforced (similarly for contact). For each set of segments, different constraints have to be enforced in combination with equilibrium, either in terms of traction or in terms of displacement discontinuity (as discussed in section 2.2).

The convergence of this outer loop is achieved when these different sets remain identical between two subsequent iterations, meaning that all the inequality constraints are satisfied.

\subsubsection{Solution of the equilibrium under constraints}

For a given set of constraints assigned to different elements, we solve for the balance of momentum combined with the corresponding prescribed set 
of equality constraints. First, we rewrite the equilibrium in terms of effective traction, such that the discretized elasticity equations (13) becomes

$$
\mathbf{t}^{\prime, n+1}=\mathbf{t}^{o}+\mathbf{E}_{\mathcal{H}} \mathbf{d}^{n+1}-\mathbf{p}_{\text {coll }}^{n+1},
$$

where $\mathbf{p}_{\text {coll }}^{n+1}=\left(0, p_{1}, 0, \ldots, 0, p_{i}, \ldots\right)$ is a vector containing the current pore pressure vector evaluated at the different collocation points, which acts only on the normal traction component.

In addition to these $4 n_{\text {elts }}$ equations, we prescribe $4 n_{\text {elts }}$ equations in relations to the type of constraint acting on each element. This results in a $8 n_{\text {elts }} \times 8 n_{\text {elts }}$ linear system with both the displacement discontinuities and the effective tractions as unknowns.

We now list the different constraints assigned to the different set of elements.

Set of elements active in tensile failure $\mathcal{S}_{a, 1}$ : Pure tensile failure is active in an element when $\digamma_{1}\left(t_{n}^{\prime, n+1}\right)>0$ and $h\left(t_{s}^{n+1}, t_{n}^{\prime, n+1}\right)<0$ at both collocation points. We thus enforce eq. (7b), and the discretized equations for one collocation point of an active tensile element are

$$
t_{n}^{\prime, n+1}=-\sigma_{c}\left(\kappa^{n+1}, \kappa_{m}^{n+}\right), \quad \Delta d_{s}=0,
$$

which can be rewritten in matrix form as

$$
\left[\begin{array}{ll}
0 & 0 \\
0 & 1
\end{array}\right]\left[\begin{array}{c}
t_{s}^{n+1} \\
t_{n}^{\prime, n+1}
\end{array}\right]=\left[\begin{array}{c}
0 \\
-\sigma_{c}\left(\kappa^{n+1}, \kappa_{m}^{n+1}\right)
\end{array}\right], \quad\left[\begin{array}{ll}
1 & 0 \\
0 & 0
\end{array}\right]\left[\begin{array}{l}
\Delta d_{s} \\
\Delta d_{n}
\end{array}\right]=\left[\begin{array}{l}
0 \\
0
\end{array}\right]
$$

Set of elements active in shear failure $\mathcal{S}_{a, 2}$. Similarly, an element for which $\digamma_{2}\left(t_{s}^{n+1}, t_{n}^{\prime, n+1}\right)>0 \quad \& \quad h\left(t_{s}^{n+1}, t_{n}^{\prime, n+1}\right) \geq 0$ at both collocation points, we must enforce $\digamma_{2}=0$ and the dilatant flow rule $(5 \mathrm{~b})$. For one collocation point of an active shear segment, we have

$$
\begin{aligned}
& t_{s}^{n+1}=c\left(\kappa^{n+1}, \kappa_{m}^{n+1}\right)+f\left(\left|d_{s}^{n+1}\right|, \delta_{m}^{n+1}\right) t_{n}^{\prime, n+1}, \\
& \Delta d_{n}=\left|\Delta d_{s}\right| \operatorname{sign}\left(t_{s}\right) \tan \left(\psi\left(\left|d_{s}^{n+1}\right|, \delta_{m}^{n+1}\right)\right),
\end{aligned}
$$

which can be rewritten in matrix form as

$$
\begin{aligned}
& {\left[\begin{array}{cc}
1 & -f\left(\left|d_{s}^{n+1}\right|, \delta_{m}^{n+1}\right) \\
0 & 0
\end{array}\right]\left[\begin{array}{c}
t_{s}^{n+1} \\
t_{n}^{\prime, n+1}
\end{array}\right]=\left[\begin{array}{c}
c\left(\kappa^{n+1}, \kappa_{m}^{n+1}\right) \\
0
\end{array}\right],} \\
& {\left[\begin{array}{cc}
0 & 0 \\
-\operatorname{sign}\left(t_{s}\right) \tan \left(\psi\left(\left|d_{s}^{n+1}\right|, \delta_{m}^{n+1}\right)\right) & 1
\end{array}\right]\left[\begin{array}{l}
\Delta d_{s} \\
\Delta d_{n}
\end{array}\right]=\left[\begin{array}{l}
0 \\
0
\end{array}\right]}
\end{aligned}
$$


Inter-penetrating segments $\mathcal{S}_{\text {interp. }}$. if the normal displacement discontinuity on one mesh node is lower than the minimum admissible value $\bar{w}_{d}$, then we enforce

$$
d_{n}^{n+1}=\bar{w}_{d} \quad \Delta d_{s}=0,
$$

which in incremental and matrix form reads

$$
\left[\begin{array}{ll}
1 & 0 \\
0 & 1
\end{array}\right]\left[\begin{array}{l}
\Delta d_{s} \\
\Delta d_{n}
\end{array}\right]=\left[\begin{array}{c}
0 \\
\bar{w}_{m}-d_{n}^{n}
\end{array}\right]
$$

Inactive elements $\mathcal{S}_{\text {inact }}$. are neither at failure or violate the inter-penetrability constraint. The rate of displacement discontinuities is zero and we enforce

$$
\left[\begin{array}{ll}
1 & 0 \\
0 & 1
\end{array}\right]\left[\begin{array}{l}
\Delta d_{s} \\
\Delta d_{n}
\end{array}\right]=\left[\begin{array}{l}
0 \\
0
\end{array}\right]
$$

\subsubsection{Solution of the tangent system for the trial active sets}

By considering all the nodes and collocation points of a computational mesh, these different constraints depending on the active set of constraints provide a set of $4 n_{\text {segm }}$ equations in addition to the elasto-static balance of momentum. We obtain the following system of $8 n_{\text {segm }} \times 8 n_{\text {segm }}$ equations

$$
\underbrace{\left[\begin{array}{cc}
\mathbf{E}_{\mathcal{H}} & \mathbf{I} \\
\mathbf{B} & \mathbf{C}
\end{array}\right]}_{\mathbf{A}} \underbrace{\left[\begin{array}{c}
\Delta \mathbf{d} \\
\mathbf{t}^{\prime, n+1}
\end{array}\right]}_{\mathbf{x}}=\underbrace{\left[\begin{array}{c}
\mathbf{t}^{o}+\mathbf{E}_{\mathcal{H}} \mathbf{d}^{n}-\mathbf{p}_{\text {coll }}^{n+1} \\
\mathbf{a}
\end{array}\right]}_{\mathbf{y}}
$$

for the unknowns increment of displacement discontinuities $\Delta \mathbf{d}$ and current effective tractions $\mathbf{t}^{\prime, n+1}$. In the system of equations $(25), \mathbf{I}$ is a $4 n_{\text {segm }} \times 4 n_{\text {segm }}$ identity matrix, a is a $4 n_{\text {segm }} \times 1$ vector that contains the right hand sides of the different equality constraints previously described. The matrix $\mathbf{B}$ and $\mathbf{C}$ are sparse and contain the constraints in term of displacement discontinuities and effective traction respectively, given by the constitutive interface relations. The pattern of these block matrices depends on the different set of constraints and thus may differ between iterations of the yielding loop.

The system of equations (25) is non-linear when the material's strength parameters soften with current plastic deformations. For this reason, we adopt a fixed point iterative scheme combined with under-relaxation [37]. Iterations are ended when subsequent estimates of both increment of displacement discontinuities and effective traction fall within a given relative 


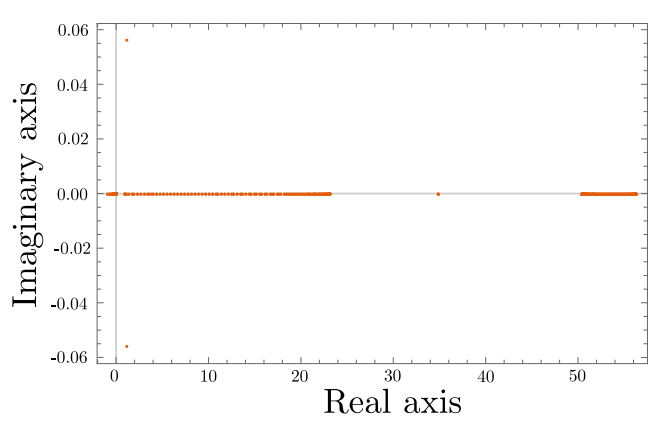

(a)

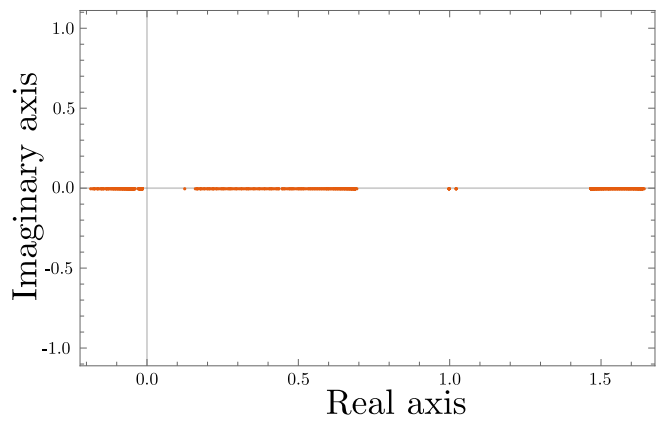

(b)

Figure 3: Examples of eigenvalues distribution along the complex plane for the matrix A that arises from final system of equations (25) prior (a) and after (b) application of preconditioning matrix $\mathbf{P}_{u p}$. Case of a planar fracture in an infinite domain discretized with 100 equal-sized segments with six element active in shear. The spectral radius of the original matrix $\mathbf{A}$ is $\rho(\mathbf{A}) \simeq 56.3$, while the one of the preconditioned matrix is $\rho\left(\mathbf{A}_{p}\right) \simeq 1.64$.

tolerance $\epsilon_{t o l}$. At a given iteration of the fixed point scheme, the solution of the system (25) is obtained via a Krylov sub-space iterative method, specifically the generalized minimal residual method (GMRES).

Although the sub-blocks $\mathbf{B}$ and $\mathbf{C}$ are singular sparse matrices, $\mathbf{A}$ has always full rank. Furthermore, although the final matrix A is sparse, it is not diagonal dominant and highly non-symmetric. Figure 3a displays an example of spectral properties of matrix $\mathbf{A}$ arising from the example of a a planar fracture embedded in an infinite medium discretized with 100 equal-sized elements, with 6 of them belonging to $\mathcal{S}_{a, 2}$, while the others being inactive. The eigenvalues of $\mathbf{A}$ are spread over a wide range on the complex plane, both along the real and the imaginary axis (Figure 3a). The spectral radius for such an example is indeed $\rho(\mathbf{A})=56.3$, resulting in a slow convergence during GMRES iterations. In order to improve the spectral properties of matrix A, we develop a block preconditioner approach. Unlike preconditioners based on algebraic techniques that require little knowledge of the problem under investigation [38], the preconditioning of system (25) is tailored to the pattern of matrix of coefficient A. Starting from the observation that if the subblock $\mathbf{C}$ is null, which is the case when all the mesh elements are inactive, the pattern of the resulting system of equations is equivalent to the one that arises from non-symmetric saddle point problems, we adapt a preconditioner that is tailored for such class of problems (see [38, 39, 40, 41] for such type 
of pre-conditioners). Following [38], we introduce an upper-triangular block preconditioner matrix on the right side of system (25) such that the latter can be rewritten as

$$
\mathbf{A P}_{u p}^{-1} \mathbf{u}=\mathbf{y}, \quad \mathbf{u}=\mathbf{P}_{u p} \mathbf{x},
$$

where the preconditioning matrix $P_{u p}$ reads

$$
\mathbf{P}_{u p}=\left[\begin{array}{cc}
\mathbf{D}_{\mathbf{E}_{\mathcal{H}}} & \mathbf{I} \\
\mathbf{0} & \mathbf{S}
\end{array}\right]
$$

and its inverse is given by

$$
\mathbf{P}_{u p}^{-1}=\left[\begin{array}{cc}
\mathbf{D}_{\mathbf{E}_{\mathcal{H}}}^{-1} & -\mathbf{D}_{\mathbf{E}_{\mathcal{H}}}^{-1} \mathbf{S}^{-1} \\
\mathbf{0} & \mathbf{S}^{-1}
\end{array}\right]
$$

In equation (27) and (28), $\mathbf{D}_{\mathbf{E}_{\mathcal{H}}}$ is the diagonal of the hierarchical elasticity matrix $\mathbf{E}_{\mathcal{H}}$ and $\mathbf{S}=\mathbf{C}-\mathbf{B D}_{\mathbf{E}_{\mathcal{H}}}^{-1}$ is the Schur complement with respect to $\mathbf{D}_{\mathbf{E}_{\mathcal{H}}}$. Note that if $\mathbf{D}_{\mathbf{E}_{\mathcal{H}}}=\mathbf{E}_{\mathcal{H}}$, then the spectrum of $\mathbf{A} \mathbf{P}_{u p}^{-1}$ is $\rho\left(\mathbf{A} \mathbf{P}_{u p}^{-1}\right)=\{1\}$ such that an iterative method like GMRES would converge in at most two iterations [38]. In practice, however, we do not want to compute the inverse of the hierarchical elasticity matrix. We consider only the inverse of the diagonal self-effect elastic contributions. It is worth mentioning that for nonsymmetric saddle point problems, this choice is commonly taken when the sub-block $(1,1)$ is diagonal dominant, for which it is proved that a good clustering of the eigenvalues around $1, \frac{1}{2}(1+\sqrt{5})$ and $\frac{1}{2}(1-\sqrt{5})$ is obtained $[38,39]$ (although it does not prevent the preconditioned matrix from having its eigenvalues on both side of the imaginary axis). Upon application of the right upper-triangular preconditioner $\mathbf{P}_{u p}^{-1}$, the system of equations (26) can be re-written as the following two systems:

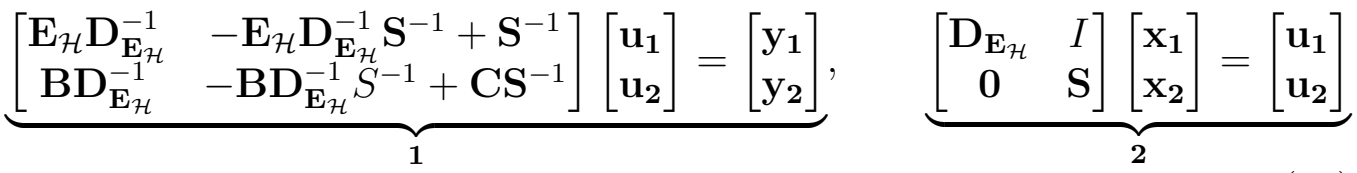

As one can notice, the exact inverse of the Schur complement is needed for numerical resolution of system 1. Although the Schur complement is a sparse matrix and fast algorithms have been developed to obtain its inverse (see $[42,43]$ for examples), its inverse is typically not sparse. For large scale problems, therefore, this operation would costly memory-wise. In order to 
avoid computing the inverse of the Schur complement $\mathbf{S}$, we perform a change of variable

$$
\mathbf{z}_{2}=\mathrm{S}^{-1} \mathbf{u}_{2}
$$

such that the system 1 of equation (29) reduces to

$$
\underbrace{\left[\begin{array}{cc}
\mathbf{E}_{\mathcal{H}} \mathbf{D}_{\mathbf{E}_{\mathcal{H}}}^{-1} & -\mathbf{E}_{\mathcal{H}} \mathbf{D}_{\mathbf{E}_{\mathcal{H}}}^{-1}+\mathbf{I} \\
\mathbf{B D}_{\mathbf{E}_{\mathcal{H}}}^{-1} & -\mathbf{B D}_{\mathbf{E}_{\mathcal{H}}}^{-1}+\mathbf{C}
\end{array}\right]}_{\mathbf{A}_{p}}\left[\begin{array}{l}
\mathbf{u}_{\mathbf{1}} \\
\mathbf{z}_{\mathbf{2}}
\end{array}\right]=\left[\begin{array}{l}
\mathbf{y}_{\mathbf{1}} \\
\mathbf{y}_{\mathbf{2}}
\end{array}\right],
$$

where $\mathbf{A}_{p}$ denotes the preconditioned matrix of coefficients. Note that, since the Schur complement and its inverse are ultimately discarded, system (30) can be found in a more direct way by directly setting $\mathbf{x}=\mathbf{P}^{-1} \mathbf{z}$ in (25), with $\mathbf{z}=\left[\begin{array}{l}\mathbf{u}_{1} \\ \mathbf{z}_{2}\end{array}\right]$ and the preconditioning matrix $\mathbf{P}=\left[\begin{array}{cc}\mathbf{D}_{\mathbf{E}_{\mathcal{H}}} & \mathbf{I} \\ \mathbf{0}_{\mathbf{I}} & \mathbf{I}\end{array}\right]$.

In order to highlight the effect of the preconditioner $\mathbf{P}_{u p}$, we show in Figure $3 \mathrm{~b}$ the spectral properties of the preconditioned matrix $\mathbf{A}_{p}$ that arises from the same example previously described. The improvement is clear. The eigenvalues of the pre-conditioned matrix are spread over a much more narrow range (see Figure 3) and more importantly all the eigenvalues are real. The spectral radius in this particular example is $\rho\left(\mathbf{A}_{p}\right) \sim 1.64$, roughly $3 \%$ of the one of the initial system A. The preconditioned system of equations (30) is solved via GMRES iterative method for the unknown vectors $\mathbf{u}_{\mathbf{1}}$ and $\mathbf{z}_{\mathbf{2}}$. Once the iterative solution converges within a given tolerance, the solution of the preconditioned mechanical problem (26) can be simply obtained by performing the proper matrix-vector multiplications, i.e.

$$
\mathbf{t}^{\prime, \mathbf{n}+\mathbf{1}}=\mathbf{x}_{\mathbf{2}}=\mathbf{z}_{\mathbf{2}}, \quad \Delta \mathbf{d}=\mathrm{x}_{\mathbf{1}}=\mathbf{D}_{\mathbf{E}_{\mathcal{H}}}^{-1}\left(\mathbf{u}_{1}-\mathbf{z}_{\mathbf{2}}\right)
$$

Note that the numerical solution of the preconditioned system (26) via a GMRES iterative scheme does never involve any matrix inversions, but only matrix-vector products.

The non-linear mechanical problem (25) converges when the relative difference between two subsequent estimates of both increment of displacement discontinuities and effective tractions fall below a given tolerance (typically $\left.10^{-6}-10^{-8}\right)$. The algorithm then moves back to the yielding loop to recheck the inequalities constraints. 


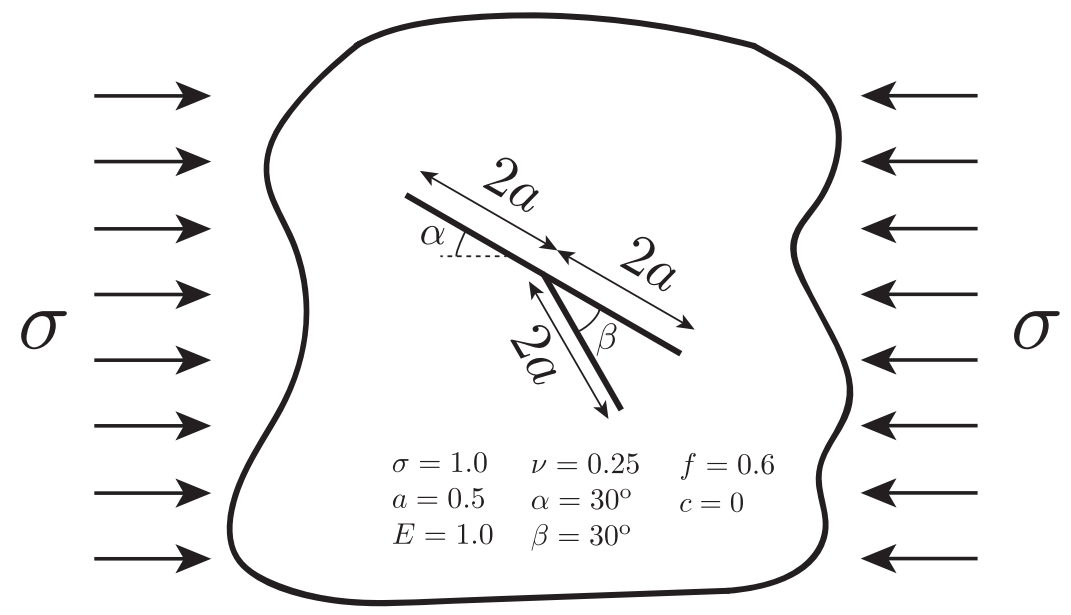

Figure 4: Sketch of branched frictional fault system subjected to a remote compressive load. All the material and geometrical parameters are reported in the figure.

\section{Illustrative examples}

\subsection{A branched frictional fault system}

As first example, we present the case of a branched frictional fault system embedded in an infinite domain and subjected to a remote static compressive load (see Figure 4 in which all material and geometrical parameters are reported). The remote load translates into applied tractions along the branched fault that are such to overcome its frictional strength and hence activate a shear crack in both branches of the system due to elastic interactions. In this example, the frictional properties are constant (no softening), cohesion as well as shear-induced dilatancy are neglected $\left(c=0, \tan \psi_{p}=0\right)$.

No analytical solution exists for this problem. We thus compare our results with previously reported numerical results for this same problem - see Maerten et al. [44] who also compare their solutions with the one of Cooke and Pollard [45]. We discretize the branched fault system with $210^{4}$ equal-sized straight segments (notably $1.210^{4}$ elements for the main branch of length $4 a$ and $0.810^{4}$ segments for the secondary branch of length $2 a$ ) for a total of $1.610^{5}$ degrees of freedom (tractions and displacement discontinuities). Using $\eta=3, \epsilon_{A C A}=10^{-6}$, and $n_{\text {leaf }}=300$, we obtain a compression ratio of $c_{r}\left(\mathbf{E}_{\mathcal{H}}\right)=0.025$ for the hierarchical matrix representation of the elastic system. This allows to solve this problem on laptop using less than 3GB of 

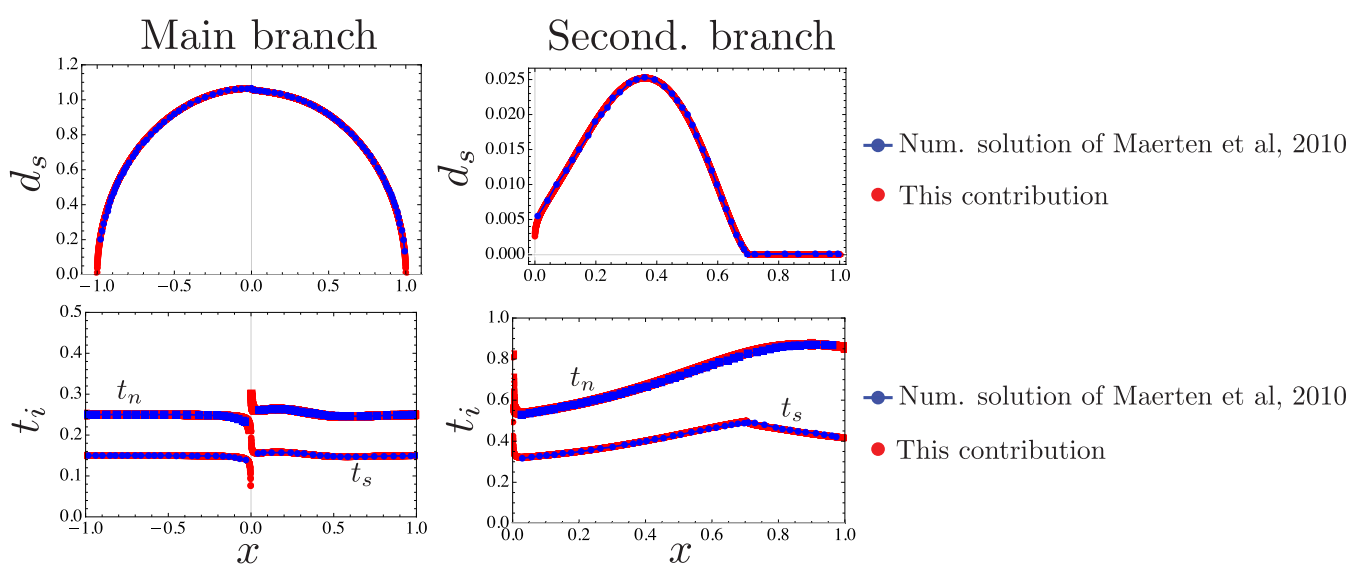

Figure 5: Comparison between the numerical results obtained with the developed solver and the ones of Maerten et al. [44] in terms of slip and tractions distribution along the main fault branch (left panel) of length $4 a$ and along the secondary branch of length $2 a$ (right panel).

RAM. It would have been impossible using the fully populated elastic matrix which requires $\sim 51 \mathrm{~GB}$ of memory storage in double precision.

The comparison of our numerical results with the one reported by Maerten et al. [44] are displayed in Figure 5. A good match between our numerical results and the ones of Maerten et al. [44] is obtained, both in terms of slip and tractions distributions. The position of the shear crack tip on the secondary branch is accurately captured, denoting thus that the algorithm devised works correctly for the frictional deformation.

\subsection{Tensile wellbore failure}

We now switch to an example associated with pure tensile failure and mode I cohesive crack initiation and growth from a wellbore located in a infinite domain (see Figure 6). We consider the case of an increase of the wellbore pressure, while the far-field in-situ stress remains constant. The material properties (large cohesion, finite tensile strength) as well as the in-situ stress field are taken to favor pure tensile failure. Upon increase of the wellbore pressure $\left(t_{n}(r=R)=p_{b}(t), \quad t_{s}(r=R)=0\right)$, a tensile fracture initiates and propagates symmetrically with respect to the centre of the wellbore along the direction of the maximum principal in-situ stress (here $\sigma_{x x}$ ). The "Kirsch" elastic solution [46] allows to estimate the wellbore pressure $p_{b, \text { strength }}=\sigma_{c}-\sigma_{x x}+3 \sigma_{y y}$ at which the hoop stress $\sigma_{\theta \theta}$ around the 


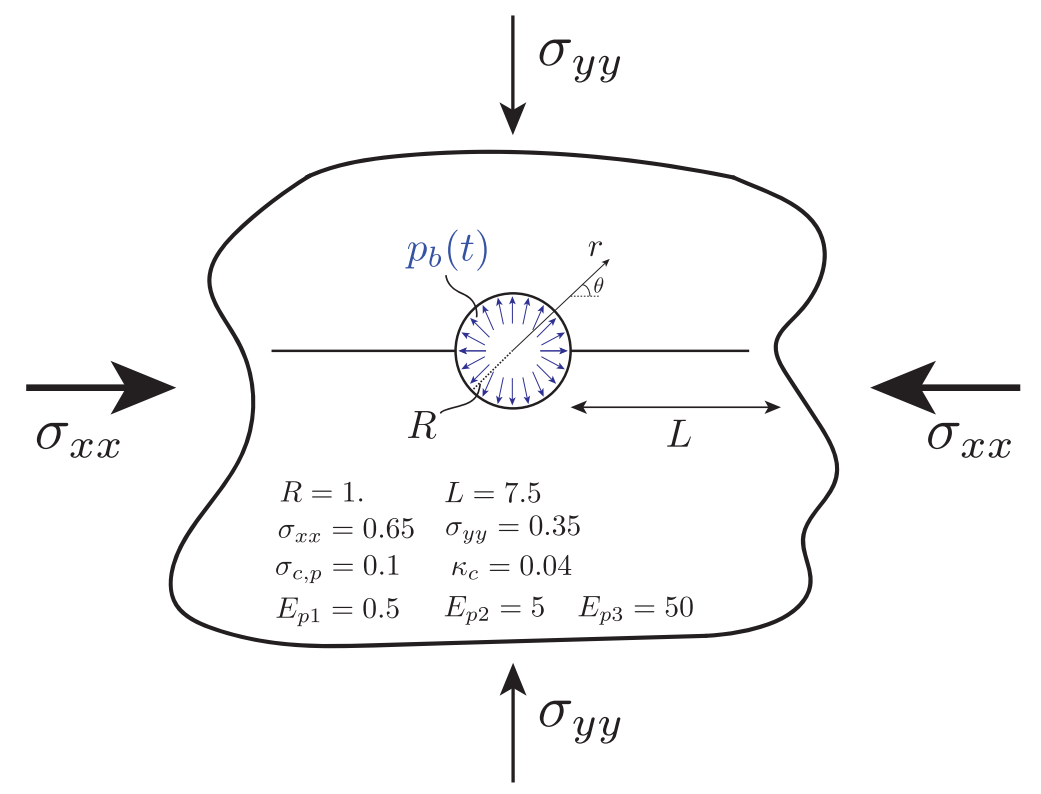

Figure 6: Sketch of plane strain pressurized wellbore \& far field loading conditions. The elasticity matrix is compressed using: $\eta=3, \epsilon_{A C A}=10^{-6}, n_{\text {leaf }}=32$ resulting in a compression ratio $c_{r}=0.1132$. We use a small value of $n_{\text {leaf }}$ here as the problem is rather small (924 elements / 3712 displacement discontinuity unknowns).

wellbore reaches the material tensile strength $\sigma_{c}$ as well as its location (here at $\theta=0$ for the given deviatoric far-field stress and $p_{b \text {,strength }}=0.5$ for the parameters of Figure 6). Due to the softening of the tensile strength, this problem exhibit a size effect on the pressure and corresponding crack length at which the crack completely nucleates. Specifically, the crack initiation pressure of the borehole is defined as the borehole pressure at which all the fracture energy has been released (or similarly at which the opening at the borehole wall equals the critical opening $\kappa_{c}$ at which cohesive forces vanishes). This initiation pressure is larger than $p_{b, \text { strength }}$ predicted from a strength criteria [47, 48]. The size effect is governed by the Irwin number defined as the ratio $\mathcal{I}$ between the material length scale $l_{m}=\frac{G_{c} E_{p}}{\sigma_{c}^{2}}$ (with $G_{c}=\frac{\sigma_{c, p} \kappa_{c}}{2}$ the critical fracture energy) and the structural length scale here the wellbore radius $l_{s}=R$. For that particular configuration large values of $\mathcal{I}$ corresponds to cases where fracture energy requirement govern crack nucleation, while strength dominated failure for low value of $\mathcal{I}$. 

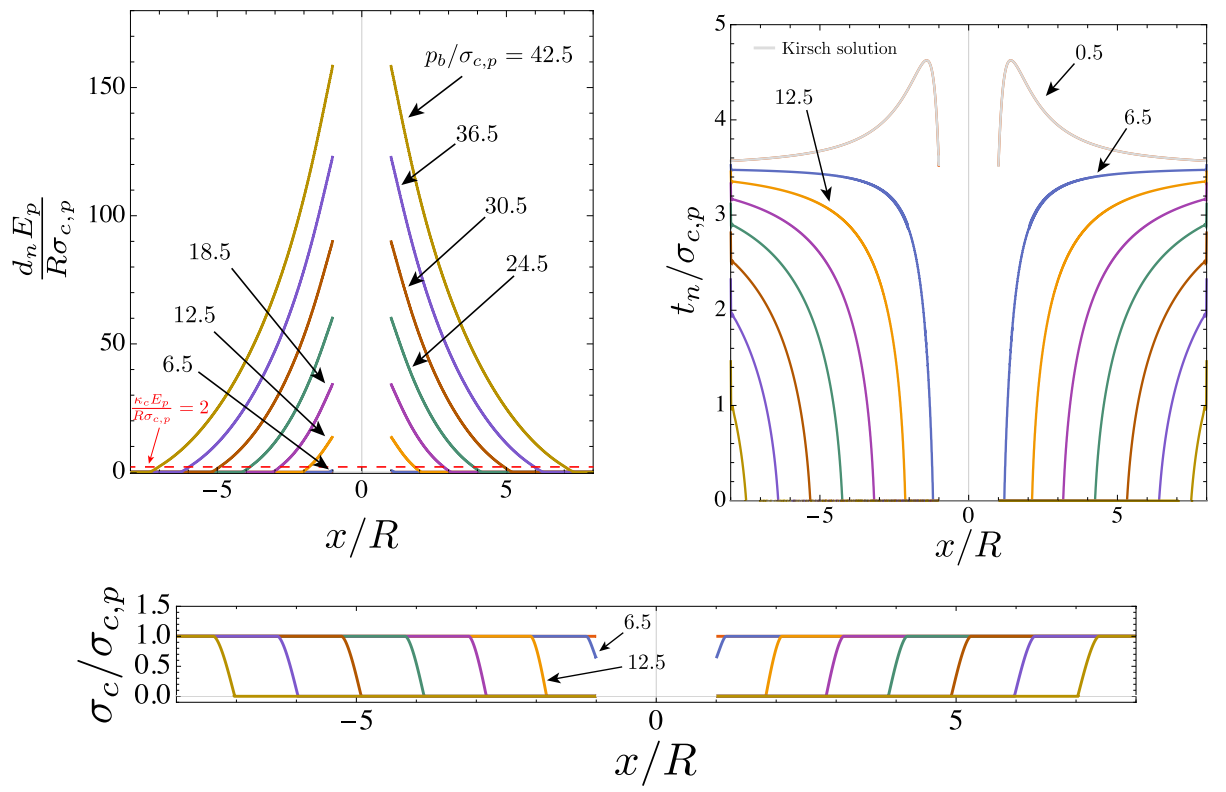

Figure 7: Spatial profiles of normalized opening displacement discontinuity $\frac{d_{n} E_{p}}{R \sigma_{c, p}}$ (topleft), normal traction $t_{n} / \sigma_{c, p}$ (top-right) and tensile strength $\sigma_{c} / \sigma_{c, p}$ (bottom) along the horizontal direction (i.e. $\theta=0$ ), at different normalized wellbore pressure $p_{b} / \sigma_{c, p}-\mathcal{I}_{2}=1$ case. The light grey lines represent the Kirsch analytical solution valid in the elastic range (prior to crack nucleation).

We perform three different simulations, varying the plane strain Young modulus $E_{p}$ to cover three distinct values of the Irwin number $\left(\mathcal{I}_{1}=0.1, \mathcal{I}_{2}=\right.$ 1 and $\mathcal{I}_{3}=10$ ) while keeping the other parameters constant (see Figure 6). In addition to the wellbore boundary, we mesh a potential horizontal line where the crack can nucleate with 924 equal-sized straight elements. Table 1 compares our numerical results to the ones reported in Lecampion [47] for the scaled crack initiation pressure for different value of $\mathcal{I}$. The results are similar within $5 \%$ relative difference.

Figure 7 displays the spatial profile of normalized opening displacement discontinuities (top-left), normal traction (top-right) and normal traction $t_{n}$ (bottom) along $\theta=0$, for increasing values of the normalized borehole pressure $p_{b} / \sigma_{c, p}$. For low values of $p_{b} / \sigma_{c, p}\left(p_{b} / \sigma_{c, p}<5\right.$ here), the response is elastic: the spatial profile of the normal traction matches perfectly the Kirsch elastic analytical solution (see the light grey line in Figure 7 top-right for $\left.p_{b} / \sigma_{c, p}=0.5\right)$. When the borehole pressure reaches the value given by 
the strength criterion (here $p_{b} / \sigma_{c, p}=5$ ), a crack starts to propagate symmetrically, and reduction of the normal tractions associated with softening can be observed in a cohesive zone near the crack tips (see Figure 7).

\subsection{Shear-banding in uniaxial compression}

The examples presented so far involved an infinite medium. However, the numerical scheme devised allows to readily investigate problems with finite domains whose boundary conditions are known a priori. Effective tractions and/or displacement discontinuities (see equations (10-11)) can thus be easily imposed through the matrices $\mathbf{B}$ and $\mathbf{C}$ in system (25). As a simple example, a bounded domain with traction-free boundary conditions must satisfy $t_{i}^{\prime}=0$ all along its boundaries. These constraints are enforced directly in matrix $\mathbf{C}$ of system (25).

We discuss now the case of a rectangular bar under plane strain conditions subjected to uni-axial compression (see Figure 8 for all geometrical and material parameters).

Our aim is to illustrate how by introducing a number of segments where localized deformation can possibly takes place, the final response of the material is akin to the one obtained with a conventional elasto-plastic approach. As a result, the mesh depicted in Figure 8 should not be confused with a finite element mesh as we use a boundary element method to solve for the balance of momentum. Indeed, the segments located inside the bar are solely here to capture localized inelastic deformation. For value of the uniaxial load below the yield stress, all the displacement discontinuities of the element inside the domain are zero and the elastic response is captured by the elements discretized the material boundary. The yield properties of all segments are taken to correspond to a purely cohesive material (zero friction and infinite tensile strength) - which translates in a Tresca material globally. We first investigate the case of perfect plasticity without softening, and then discuss the effect of softening.

In absence of softening, the elasto-plastic response for such a configuration yields homogeneous plastic deformation in the case of a "defect" free homogeneous material. An elastic perfectly plastic solid with smooth yield surface is indeed quite resistant to localization of deformation into a shear band $[49,50]$. However, small heterogeneities in strength typically results in localization of deformation into shear bands. This is notably the case when a "defect" is introduced in the middle of the bar - see [3, 51, 4, 52] for discussion of the uni-axial tension case. 


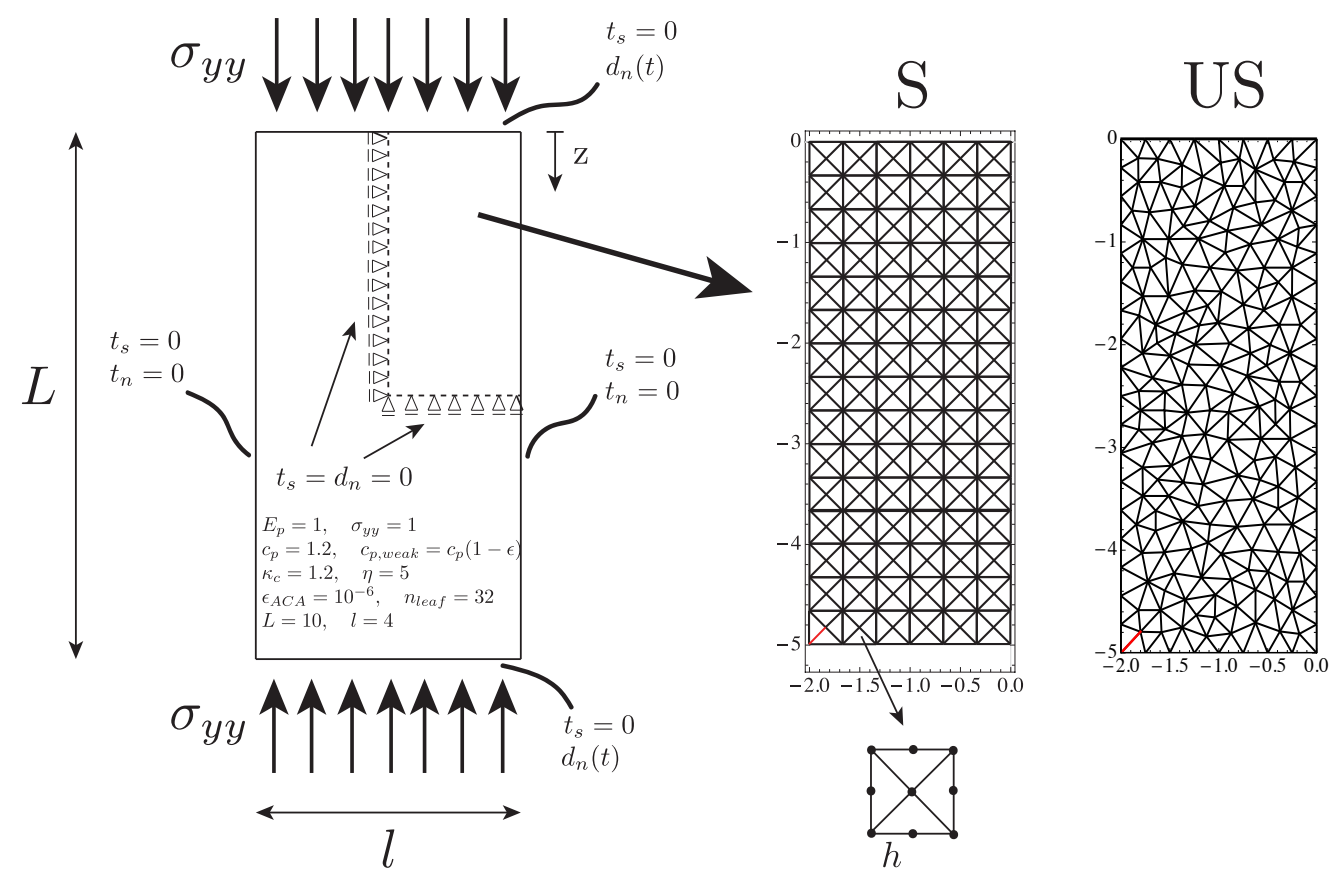

Figure 8: A rectangular bar subjected to uni-axial compression. Only one quarter of the bar is modelled due to symmetry. A set of structured (S) or unstructured (US) potential segments of failures are tested to investigate the corresponding mesh dependency. A defect (segment with lower strength) is introduced near the bottom-left corner (red segment).

In order to investigate the mesh dependency and the intrinsic limits/advantages of our method, we solve the problem using two computational boundary element meshes (see Figure 8-right): i) a structured mesh (S), for which the potential failure segments for plasticity localization follow a specific geometrical pattern which includes the preferential $45^{\circ}$ direction for a Tresca material, and ii) an un-structured mesh (US) whose potential failure segments are randomly oriented in the problem domain. We introduce a defect at the bottom-left corner of the bar by reducing the frictional strength of the extreme bottom-left segment such that (see red segment in Figure 8-right)

$$
c_{p, \text { weak }}=c_{p}(1-\epsilon),
$$

where $\epsilon$ is a dimensionless parameter that quantify the intensity of the defect. The uniform compression within the bar is increased by prescribing increasing the normal displacement discontinuities of the top surface. 


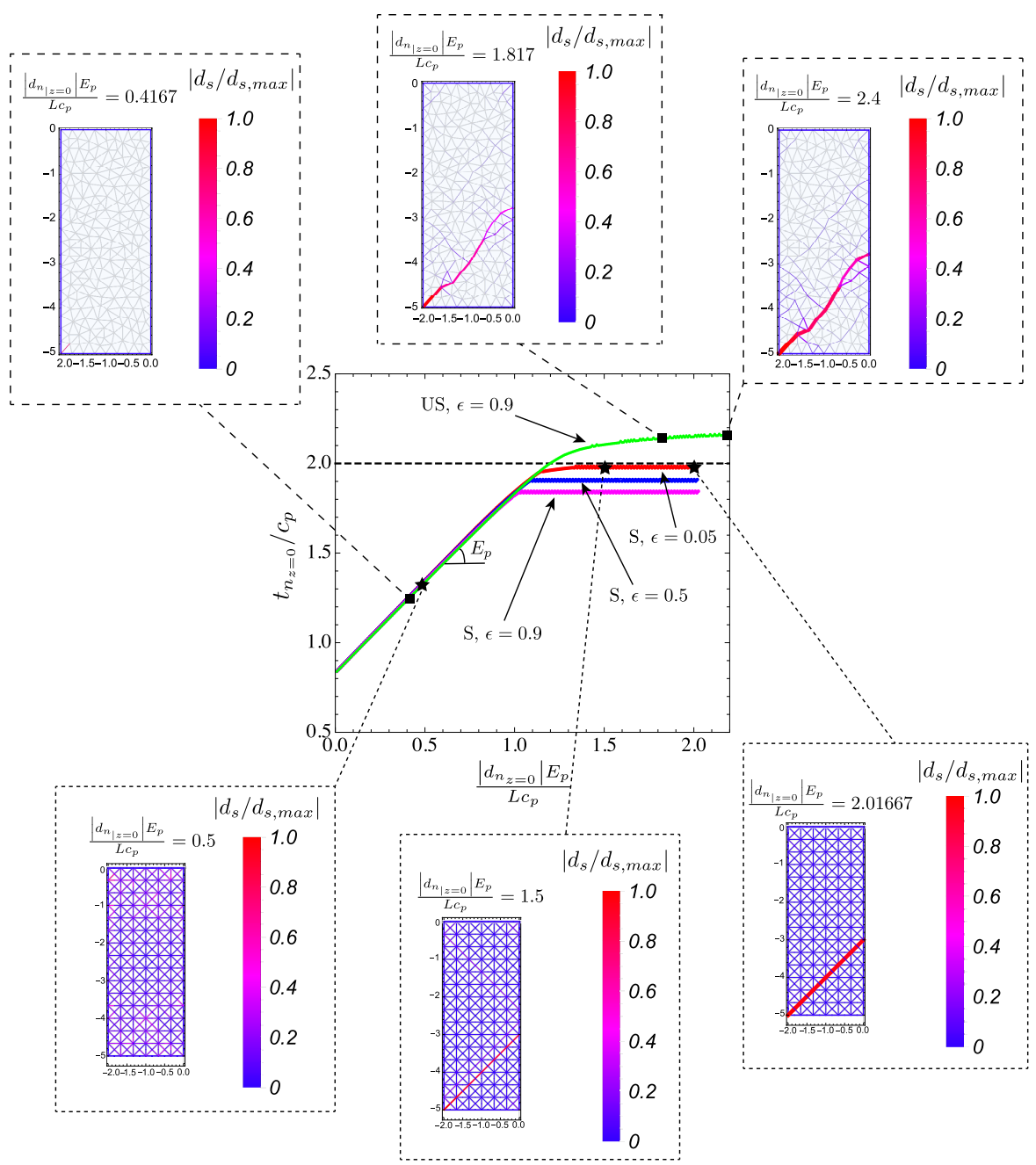

Figure 9: Load-displacement curves for both structured (S) and unstructured (US) mesh, for different intensity values of in-homogeneity $\epsilon$ (center plot). The horizontal dashed black line represents the (normalized) traction value at $z=0$ for plasticity nucleation that one would get if an homogeneous bar with only a pre-meshed slip line at $45^{\circ}$ is considered (from the bottom-left corner of the bar to the tractions free lateral side). Evolution of normalized plastic shear deformations $\left|d_{s} / d_{s, \max }\right|$ along pre-existing potential failure segments (structured and unstructured mesh) is displayed at different moment along the stress-strain curve. The color and the thickness of each pre-existing segment is proportional to the corresponding shear displacement discontinuity accumulated. for both structured (S) and unstructured (US) mesh for different intensity 
values of in-homogeneity $\epsilon$, without any softening of cohesion. Although the material response is qualitatively the same regardless the type of mesh and the intensity of the defect, the level of compression at which a shear band is nucleated is not mesh independent.

Referring to the case of structured mesh with $\epsilon=0.05$, the load-displacement (using the normalized displacement at the top of the bar $\frac{\left|d_{n_{\mid z=0} \mid}\right| E_{p}}{L c_{p}}$ ) response of the material for compression values lower than $\sim 1.05$ is perfectly linearelastic. For increasing values of compression, shear plastic deformations first take place near the inhomogeneity, up to a given value of compression after which a main shear band is triggered, from the bottom-left corner to the right side of the bar with an inclination of $45^{\circ}$ with respect to the minimum principal direction. At this specific value of compression, a small increase of compressive normal stress leads to a large increment of inelastic deformations. Localized shearing along a favourably oriented plane occur and the intensity of slip accumulated increases significantly (see the snapshots for $\frac{\left|d_{n_{\mid z=0}}\right| E_{p}}{L c_{p}}=1.5 / 2.016$ in the bottom-right of Figure 9). Because of the structured mesh adopted (that embeds the theoretical failure line of the shear band) and the low value of inhomogeneity used in this example, the nucleation of the shear band occurs at a compression value that is slightly below the theoretical value of $2 c$ that one would get if an homogeneous bar with only a pre-meshed slip line at $45^{\circ}$ is considered (see horizontal dashed black line in Figure 9-plot in the centre). This picture, however, changes for defects with larger intensities (i.e. larger $\epsilon$ ) or when an unstructured mesh is used. In the former case, larger stress concentrations near the bottom-left corner of the bar promote the nucleation of a shear band at lower values of compressive stress (as expected - see plot in the centre of Figure 9), whereas the material response in the case of the unstructured mesh is clearly stiffer (compared to the one of the structured mesh, for the same value of inhomogeneity - see the green curve in the centre plot of Figure 9), leading to a shear band nucleation at larger values of compression. This latter scenario is the result of a mesh dependency that kicks in when the pre-existing potential failure segments are not exactly aligned along the actual theoretical failure plane.

For specific problems that involve shear band localization along known 


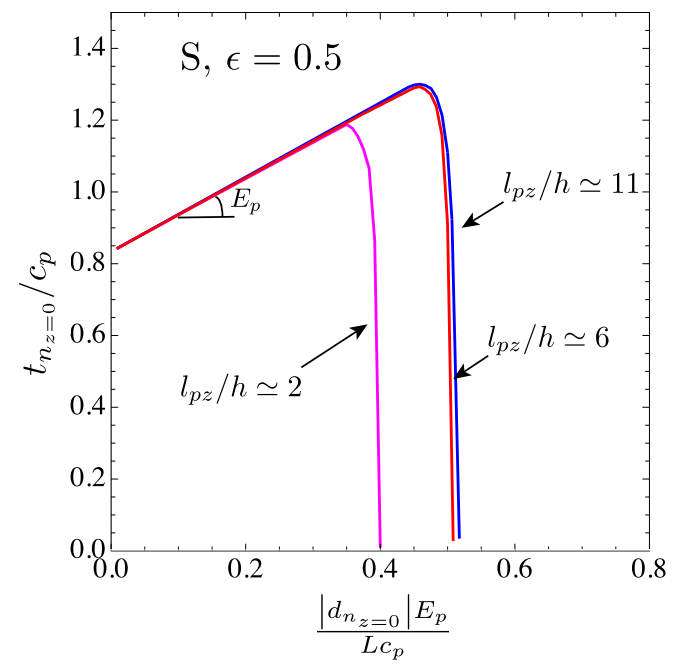

Figure 10: Softening case - Load-displacement curves for a bar subjected to uni-axial compression, discretized with a structured mesh (S) with an initial defect of intensity $\epsilon=0.5$. Effect of the mesh size $h$ as function with respect to the softening material length scale $l_{p z}=\frac{E_{p} \kappa_{c}}{c_{p}}$.

failure planes, the numerical solver introduced in section 3 is mesh independent upon meshing the a-priori known failure plane(s) with potential failing segments. More interestingly, the introduction of softening (which typically strongly re-inforce mesh dependency when using bulk elasto-plasticity) does not alter this conclusion as long as the softening material length scale $l_{p z}=\frac{E_{p} \kappa_{c}}{c_{p}}$ is properly captured numerically. This is clearly seen in Figure 10, where the load-displacement curve for the structured mesh with an inhomogeneity of $\epsilon=0.5$ is reported for different ratio of $l_{p z} / h$ being $h$ the element size. For a number of elements within $l_{p z}$ larger than $\sim 5$, the loaddisplacement curves are similar both in the linear elastic and in the softening plastic range.

\subsection{Active Earth pressure against a rigid retaining wall}

As another example of interior problem, we present the case of a retaining wall under plain strain conditions, subjected to active Earth pressure (see sketch in Figure 11-top). We assume that the retaining wall is rigid and perfectly smooth (zero friction between the soil and the wall). We assume a 

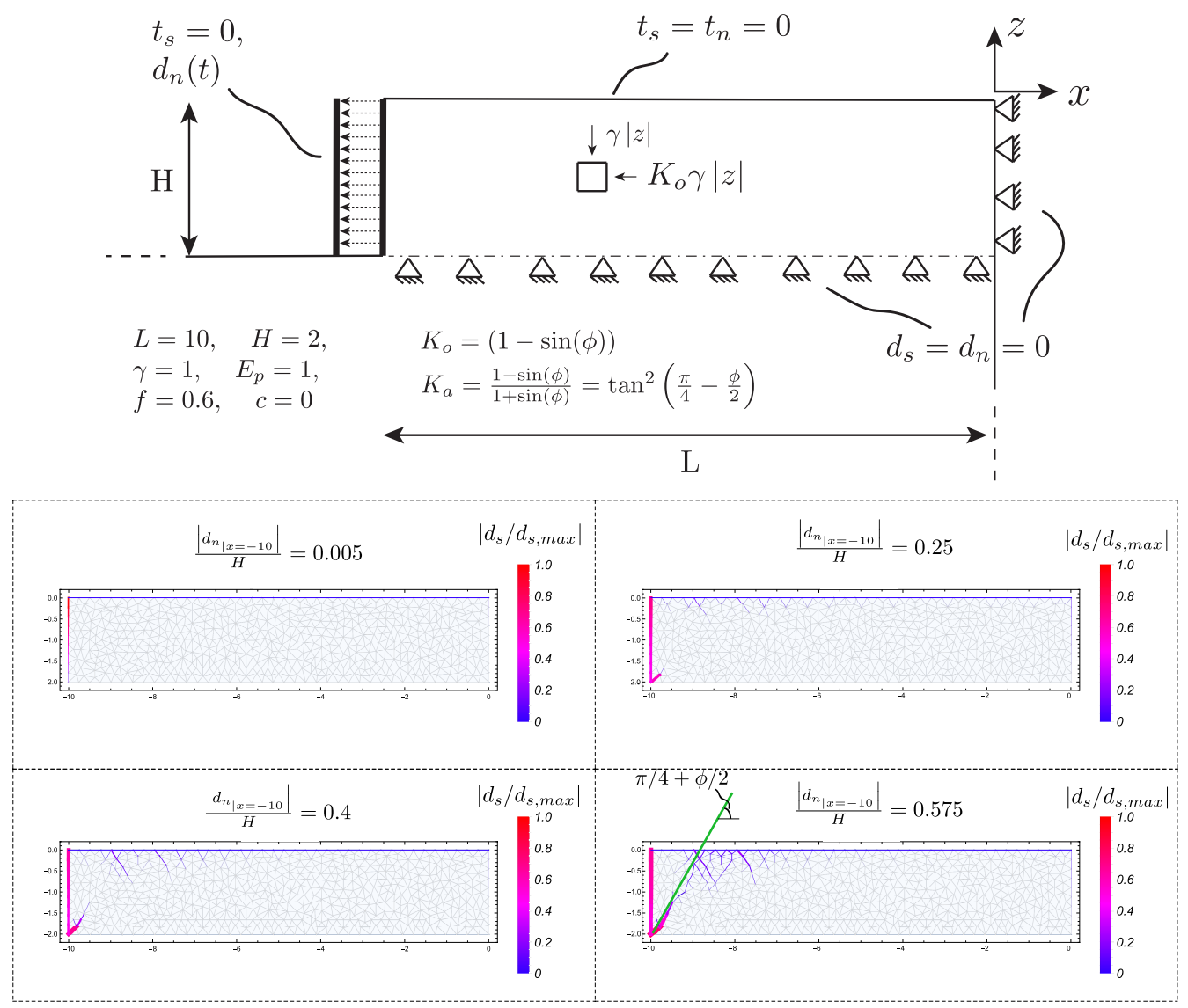

Figure 11: Top: sketch of a retaining wall \& boundary conditions adopted. Bottom: evolution of normalized plastic shear deformations $\left|d_{s} / d_{s, \max }\right|$ along the pre-existing potential failure segments (unstructured mesh) as a function of normalized translation of the rigid wall $\left|d_{n_{\mid x=-10}}\right| / H$.

purely frictional material with zero cohesion. At initial conditions, the stress state is given by two compressive principal stresses: the vertical stress $\gamma|z|$ due to the soil weight and the horizontal stress $K_{o} \gamma|z|$ due to the lateral confinement with $K_{o}=(1-\sin (\phi))$ the coefficient of Earth pressure at rest and $\phi=\arctan (f)$ the internal friction angle of the material. The limit active state is reached by reducing the horizontal principal stresses, while keeping the vertical stress constant, until their ratio equals the active Earth pressure 


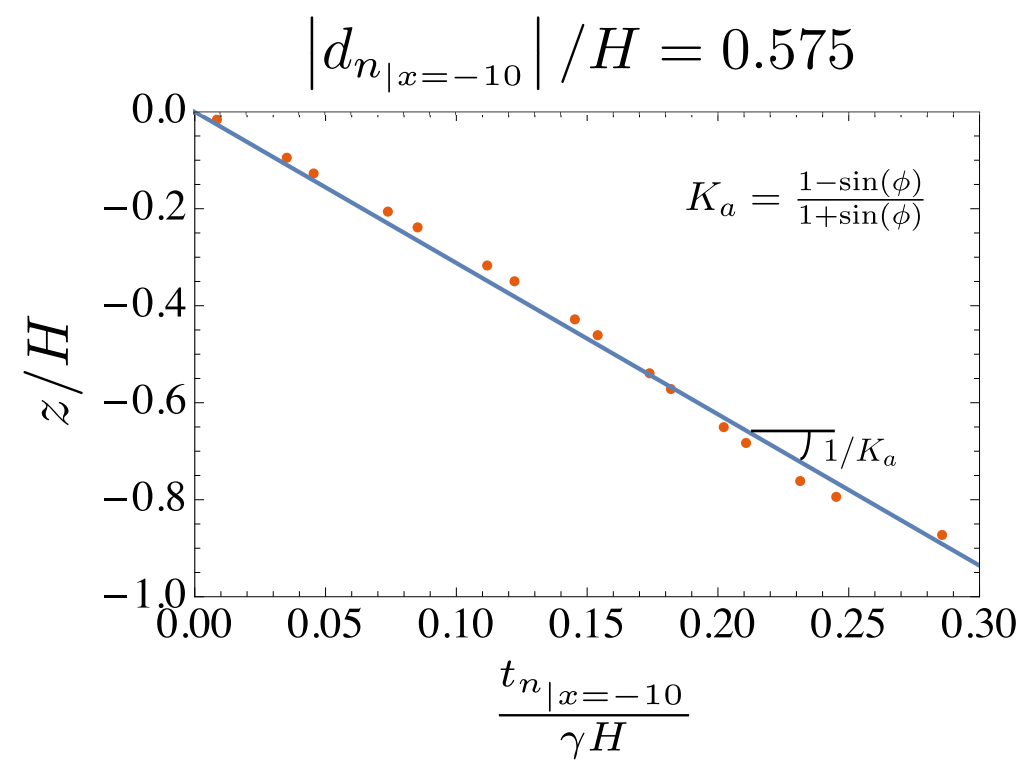

Figure 12: Vertical profile of normalized horizontal stress distribution along the retaining wall (i.e. at $x=-10$ ) in corresponding of an active limit state. The blue solid line corresponds to the theoretical solution from Rankine theory $\left(K_{a}=\frac{1-\sin (\phi)}{1+\sin (\phi)}\right)$.

coefficient $K_{a}$ (obtained from Rankine theory [53])

$$
K_{a}=\frac{1-\sin (\phi)}{1+\sin (\phi)}=\tan ^{2}\left(\frac{\pi}{4}-\frac{\phi}{2}\right)
$$

Numerically, this is obtained by translating the rigid wall along the horizontal direction by prescribing a constant normal displacement discontinuities along the wall while imposing zero shear stress at the wall (see Figure 11-top for geometry, input data and boundary conditions of the problem).

Figure 11-bottom displays the evolution of cumulative plastic shear deformations within the soil as function of the normalized lateral displacement of the wall, until the active state is reached. The progressive decrease of lateral confinement associated with the translation of the wall leads to progressive plastic failure that starts to develop from the bottom-left corner, where the stress concentration is higher, and moves up to the traction free surface. Although the progressive failure path is not straight due to the unstructured mesh of potential failing segments used, its approximate angle with respect to the minimum principal direction during active limit state is very close to the 
theoretical value from Rankine theory $\pi / 4+\phi / 2$ (see Figure 11-bottom). The horizontal stress distribution along the wall is also following the theoretical prediction $\sigma_{x x}=K_{a} \sigma_{z z}=K_{a}(\gamma|z|$ ) (see Figure 12).

\subsection{Fluid injection into a frictional weakening planar fault}

The numerical solver described in section 3 is capable of solving one-way coupled hydro-mechanical problems, where the pore-pressure history is obtained from a flow solver. As a first example, we investigate the case of fluid injection into a frictional weakening planar fault in an infinite and impermeable medium. The fault is subjected to an initial uniform effective stress state with normal and tangential component denoted respectively as $\sigma_{o}^{\prime}$ and $\tau_{o}$. In this example, the fault is characterized by a constant longitudinal permeability $k_{f}$. The friction coefficient $f$ of the fault is supposed to soften linearly with shear slip from a peak value $f_{p}$, up to a residual value $f_{r}$ at large deformations. Fluid is injected at a point under constant over-pressure $\Delta P$ (above the initial pore pressure $p_{o}$ ) with the purpose of activating slip upon local violation of the shear weakening Mohr-Coulomb yielding criterion (no cohesion $c=0$ ). This specific problem has been solved by Garagash and Germanovich [54] semi-analytically. In order to test the accuracy of our numerical solver with a time-dependent, one-way coupled and non-linear hydro-mechanical problem, we discretize the fault plane with $10^{3}$ equallysized straight segments. We vary the compression of the fully populated elasticity matrix by using four values of $\eta=0,0.1,0.8,3$, obtaining respectively compression ratios of $c_{r}=1,0.296,0.123$ and 0.093 (for $\epsilon_{A C A}=10^{-4}$ and $n_{\text {leaf }}=16$ ). Furthermore, we ensure that all the simulations follow the exact same time-steps evolution so as to calculate a relative difference at each time step with the results obtained without using a hierarchical matrix approximation ( $\eta=0$ that we take as reference numerical solution).

Figure 13 displays the time evolution of normalized half-crack length (left) and the peak slip accumulated at the middle of the fault (right), for the case of a marginally pressurized fault $\tau_{o} / \tau_{p}=0.55$ where $\tau_{p}=f_{p}\left(\sigma_{o}-p_{o}\right)=f_{p} \sigma_{o}^{\prime}$ is the peak shear strength of the fault at ambient conditions, moderate injection overpressure $\Delta P / \sigma_{o}^{\prime}=0.5$ and the coarser hierarchical approximation of the elasticity matrix $\eta=1$. The numerical results are in very good agreement with the ones of Garagash and Germanovich [54], both for the evolution of the shear crack length as well as the peak slip at $x=0$. The aseismic crack propagation is followed by the nucleation of a dynamic rupture and an arrest related to the shear crack catching up the fluid front (see Garagash and 

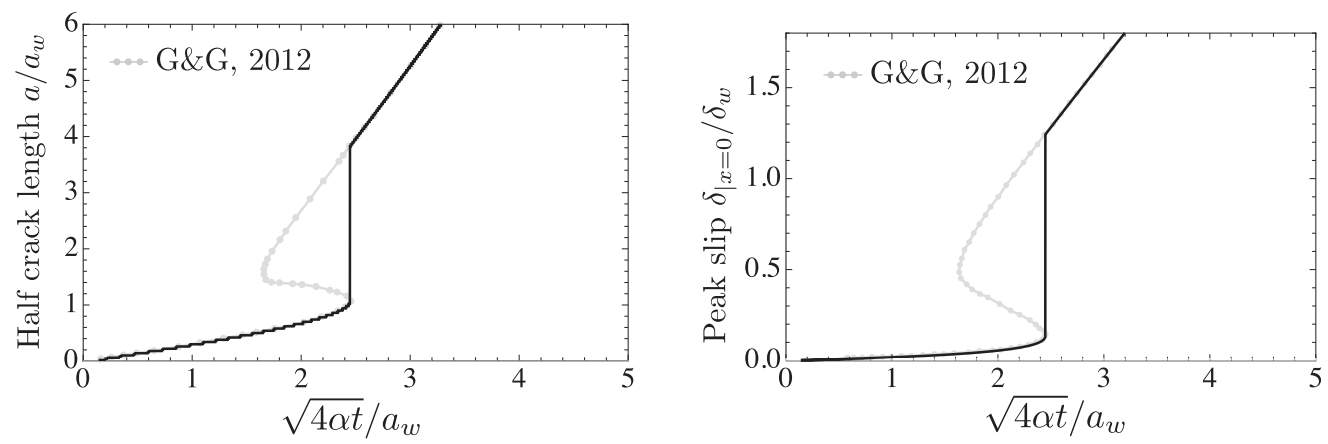

Figure 13: Time evolution of the normalized half-crack length $a / a_{w}$ (left) and normalized peak slip $\delta / \delta_{w}$ at the middle of the fault (right), i.e. at $x=0$, for an ultimately stable fault $\left(\tau_{o} / \tau_{p}=0.55\right)$, subjected to a moderate over-pressure $\Delta P / \sigma_{o}^{\prime}=0.5 . a_{w}$ and $\delta_{w}$ are the characteristic patch length and slip weakening scale, respectively (see [54] for details). The friction weakening ratio considered is taken here as $f_{r} / f_{p}=0.6$.

Germanovich [54] for discussion). This non-trivial evolution is well captured by our numerical solver. In table 2 , we report the maximum relative difference in terms for the half crack length and peak slip at $x=0$ obtained during their time evolution (taking the numerical results for the non-approximated elasticity matrix as a reference). Even for large compression, the relative error never exceeds $1.2 \%$, showing a good accuracy and a significant computational gain. For a GMRES tolerance equal to $10^{-8}$, the comparison of total CPU times (scaled by the total CPU time for the uncompressed case $\eta=0$ ), shows that the use of a hierarchical matrix approximation leads to nearly a $\sim 5$-fold speed-up with respect to the uncompressed case. These results have been obtained using a C++ implementation of the numerical solver, running on a computer with Intel Core i5 @ $2.9 \mathrm{GHz}$.

\subsection{Fluid injection in a critically stressed fractured rock mass}

As a final example, we present the case of a hydraulic stimulation of a fractured rock mass, subjected to a compressive far-field stress state with an effective principal components denoted by $\sigma_{x x}^{o}$ and $\sigma_{y y}^{\prime o}$ (with $\sigma_{x x}^{\prime o}>\sigma_{y y}^{\prime o}$ ). In this example, failure can localize only along a set of 251 randomly oriented pre-existing fractures, which are uniformly located within the region of interest $L \times L$. We adopt a power law distribution for fracture length generation with cut-off for minimum and maximum fracture lengths. This choice has been demonstrated in numerous studies at different scales and in different tectonic setting $[55,56]$. 


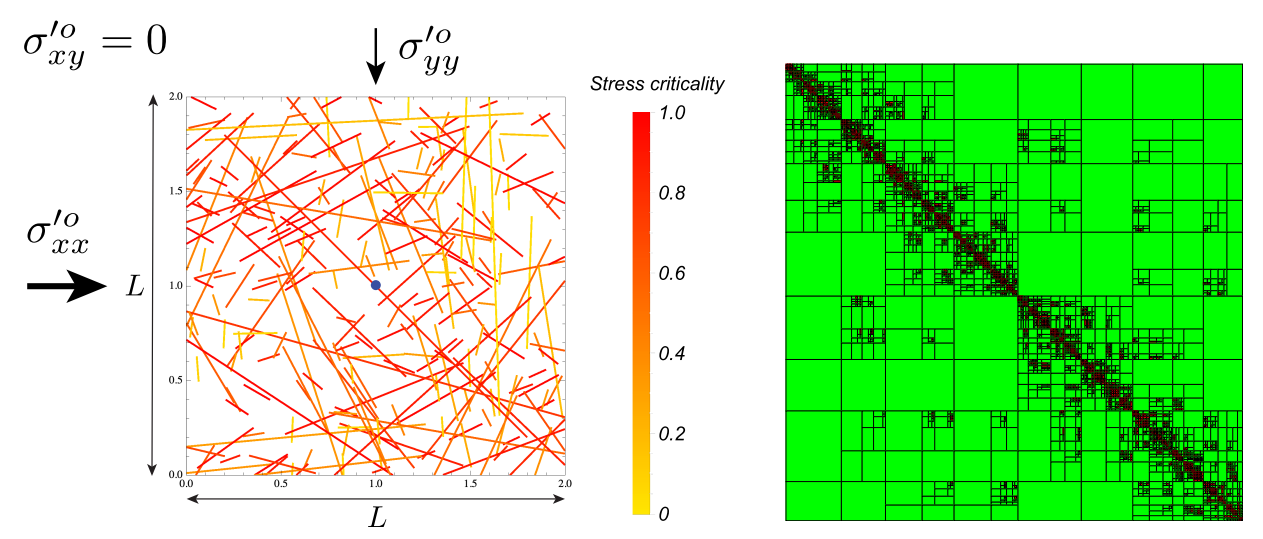

Figure 14: Left: critically stressed discrete fracture network. The color of each fracture denotes the stress criticality $\Lambda$ at ambient condition. Right: hierarchical matrix pattern upon compression (with $\eta=3, \quad \epsilon_{A C A}=10^{-6}$ and $n_{\text {leaf }}=100$ ) with low-rank block in green.

Upon generation of 251 pre-existing fractures within the elementary area of characteristic size $L$ (see Figure 14-left) and discretization with 11376 straight segments resulting in a total of $\sim 10^{5}$ unknowns, fluid is injected at $(L / 2 ; L / 2)$ under a constant injection over-pressure $\Delta P$ (in excess of the initial pore pressure $p_{o}$ ) such that it always remain below the minimum principal effective normal stress (to avoid tensile opening of any fractures). We assume that the permeability of the host medium is much smaller than the one of the fracture such that the fluid flow only within the pre-existing fractures (characterized by constant hydraulic diffusivity $\alpha$ ). The fluid flow is solved via a finite volume solver - uncoupled here to the mechanical deformation. The pre-existing fractures exhibit a purely frictional behavior with zero cohesion and without any softening (constant friction coefficient $f=0.6$ ).

We scale all the spatial variables with $L / 2$, which is the minimum distance that the fluid front can 'travel' before reaching the boundary of the region of interest (supposing that fluid is injected at $(L / 2 ; L / 2)$ ) and the time $t$ with the characteristic fluid diffusion timescale $L^{2} /(16 \alpha)$. The characteristic scales for fluid over-pressure $\Delta p=\left(p-p_{o}\right)$ and effective tractions $t_{i}^{\prime}$ are respectively the in-situ effective normal traction $t_{n, o}^{\prime, k_{i n j}}$ and the peak shear strength $t_{s, p}^{k_{i n j}}=f \cdot t_{n, o}^{\prime, k_{i n j}}$ of the fracture in which fluid is injected into, while shear slip is scaled using the characteristic scale $d_{s, w}=\frac{t_{s, p}^{k_{i n j}}}{E^{\prime}} \frac{L}{2}$ that derives from elasticity. Upon scaling the governing equations with the previous 
characteristic scales, the solution given by $\left(p-p_{p}\right) / t_{n, o}^{\prime, k_{i n j}}, t_{i}^{\prime} / t_{s, p}^{k_{i n j}}, d_{s} / d_{s, w}$ is function of (besides the geometry of the pre-existing fractures network) two dimensionless parameters: i) a stress criticality ratio

$$
\Lambda=\frac{(\kappa-1)}{f} \frac{\operatorname{Cot}(\theta)}{\left(\kappa \operatorname{Cot}(\theta)^{2}+1\right)},
$$

which is function of effective stress anisotropy ratio $\kappa=\frac{\sigma_{x x}^{\prime o}}{\sigma_{y y}^{\prime o}}$, friction coefficient $f$ and local fracture orientation $\theta$ with respect to the minimum principal direction, and ii) normalized injection over-pressure at fracture $k_{i n j}$ in which fluid is injected into

$$
\Pi=\frac{\Delta P}{t_{n, o}^{\prime, k_{i n j}}},
$$

where $t_{n, o}^{\prime, k_{i n j}}$ is the uniform ambient effective normal stress along the fracture $k_{i n j}$. Because of the relatively large effective stress anisotropy ratio $\kappa=3$ used in this example, all the pre-existing fractures oriented along the critical angle $\theta_{c}=\pi / 4+\phi / 2$ are critically stressed (see Figure 14-left). They are prompt to fail with little pressurization.

Due to the large number of unknowns, we use a hierarchical approximation of the elasticity matrix using $\eta=3, \quad \epsilon_{A C A}=10^{-6}, \quad n_{\text {leaf }}=100$ resulting in a a compression ratio of $c_{r}=0.0751$, sufficient to be able to run the simulation with a $2.9 \mathrm{GHz}$ Intel Core i5 laptop with $8 \mathrm{~GB}$ memory (see the pattern of hierarchical matrix in Figure 14-right).

As one can see from Figure 15 that displays the normalized over-pressure and shear rupture evolution in function of normalized time/fluid front position, right after fluid injection the slipping patch evolves rapidly, much faster than fluid diffusion front. As the slipping patch propagates, the stress state changes within the elementary area, activating other fractures. At a normalized time $\frac{\sqrt{4 \alpha t}}{L / 2} \simeq 0.37$, the pressurized zone is still confined to the surrounding of the injection point, while the slipping patch is significantly larger. The slipping patch evolution is thus mainly driven by stress interaction between active fractures.

The numerical solver devised captures well the yielding evolution driven by fluid flow and elastic stress interactions between activated pre-existing fractures. That example with $\sim 10^{5}$ degrees of freedom demonstrates the robustness of the preconditioning developed in section 3 . The number of 


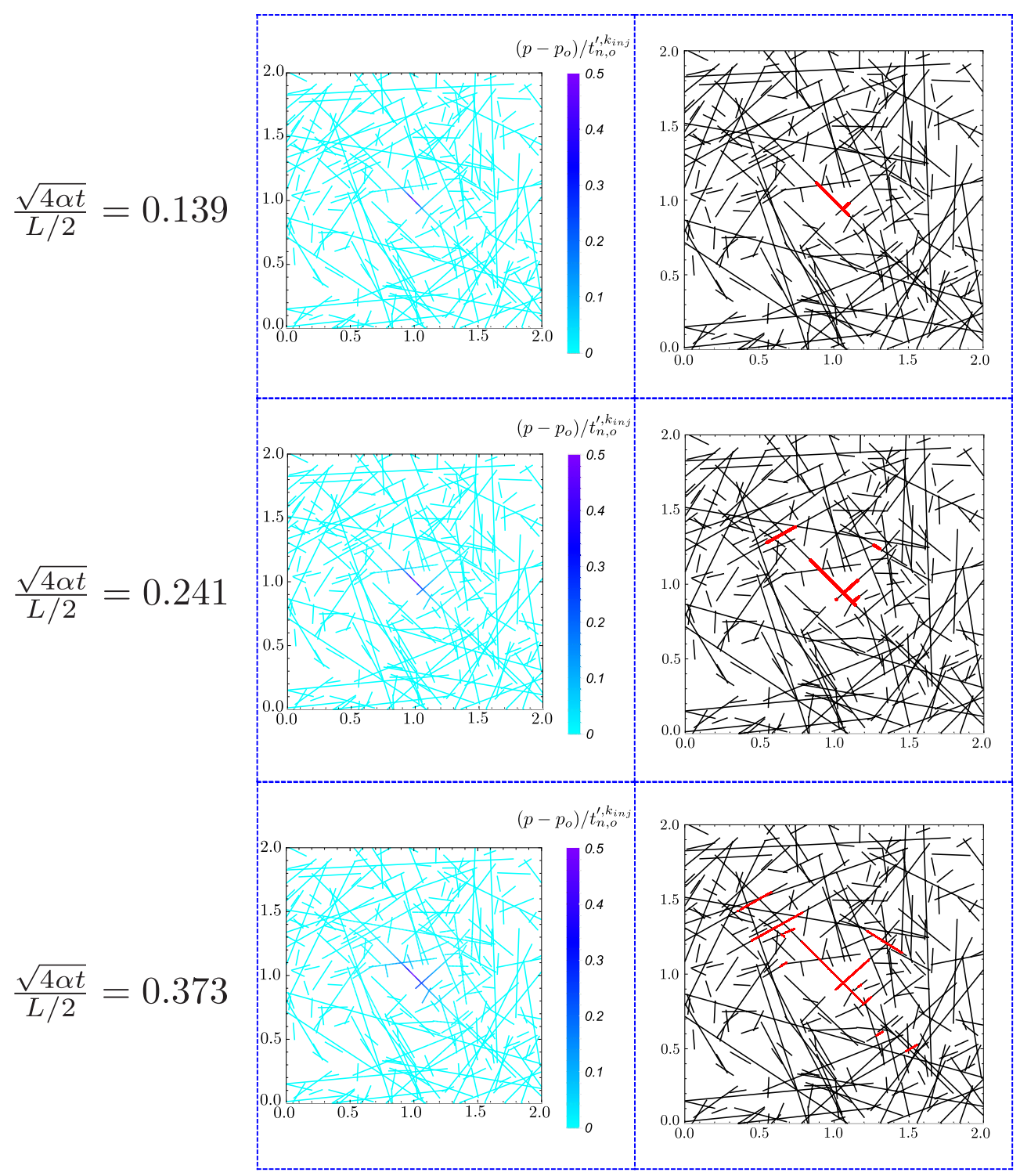

Figure 15: Evolution of normalized over-pressure (left column) and plasticity localization (shear deformations - right column) along the pre-existing critically stressed fracture network in function of normalized time/fluid front position $\frac{\sqrt{4 \alpha t}}{L / 2}$. Fluid is injected at moderate over-pressure $\Delta P / t_{n, o}^{\prime, k_{i n j}}=0.5$ into one fracture that intersect the injection point located at $(1,1)$. 


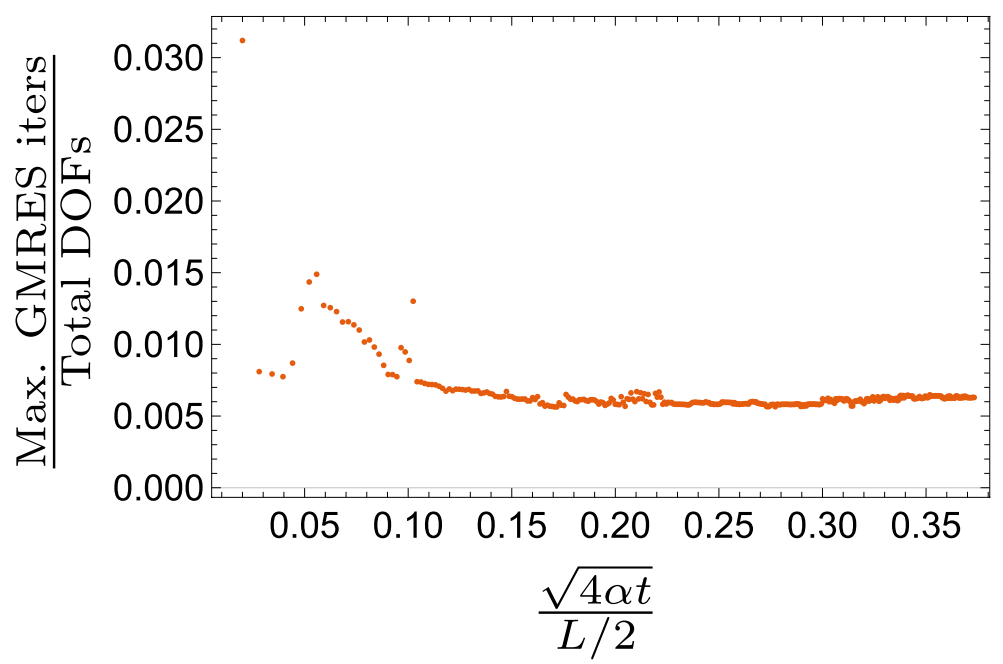

Figure 16: Maximum number of GMRES iterations required to solve the mechanical problem at each time step. A convergence tolerance $t o l=10^{-6}$ was used for the GMRES iterative solver.

iterations (scaled by the number of unknowns) for the GMRES iterative solver remains below 1.5\% for all time steps as can be seen in Figure 16.

\section{Conclusions}

We have presented a new boundary element based formulation for inelastic localized deformation along potential pre-existing failure planes. The Mohr-Coulomb criteria combined with a tensile cut-off and the linear softening laws used here can easily be replaced by more refined constitutive models if necessary. The efficiency of the numerical scheme devised rely on the use of i) a hierarchical approximation of the elastic influence matrix and ii) a block pre-conditioner specifically developed here. The proposed computational method shares similarities with the intrinsic cohesive zone element approach used in the FEM context where cohesive elements are activated upon yielding at the interfaces between finite elements $[18,19,10]$. However, the use of a boundary element method allows to decouple the discretization of the failure plane and the rest of the medium (whose elasticity is built-in BEM). This is particularly attractive for problems in infinite domain as well as cases where deformation is strongly localized into a finite number of shear bands or cracks. The approach is also advantageous when fluid flow and me- 
chanical deformation are coupled such as for hydraulic fracturing problems [57]. With respect to the hydro-mechanical behavior of fractured rocks, it would be interesting to further compare the approach proposed here (which explicitly model all discontinuities) with continuum non-local microstructural brittle damage models $[58,59]$.

The different examples reported demonstrate the versatility of the proposed approach in dealing with various problems exhibiting localized plastic deformation as well as crack growth. Unlike others existing BEM or FEM formulations for inelastic problems based on bulk plasticity with softening, this numerical scheme does not show mesh dependency as long as the softening length-scale is properly resolved and -more importantly- that the true plane of localized deformations are discretized (in other words known a-priori). This last point can be fixed by modifying/refining the discretization of the initial DD segments in an adaptive manner according to a measure of inelastic deformation (e.g. shear dissipation) averaged in the bulk. Another possible extension of the proposed algorithm is to move to an approach where new DD elements are added in the proper direction to capture the plane of localized deformation as it progresses. Such an algorithm would require to search iteratively for direction of failure advancement ahead of the shear-bands/cracks using a similar yielding criteria.

\section{Acknowledgements}

This work was funded by the Swiss National Science Foundation (grant 160577) and the Swiss Federal Office of Energy (grant S/I 50135401).

\section{Authors contributions}

Federico Ciardo: Conceptualization, Methodology, Formal analysis, Investigation, Software, Visualization, Validation, Writing - original draft

Brice Lecampion: Conceptualization, Methodology, Formal analysis, Investigation, Writing - review \& editing, Supervision

François Fayard: Investigation, Software, Validation

Stéphanie Chaillat: Conceptualization, Software, Writing - review \& editing

\section{References}

[1] A. Needleman, A numerical study of necking in circular cylindrical bars, J. Mech. Phys. Solids 20 (1972) 111-127.

[2] A. Needleman, V. Tvergaard, Necking of biaxially stretched elasticplastic circular plates, J. Mech. Phys. Solids 25 (1977) 159-183. 
[3] V. Tvergaard, A. Needleman, K. K. Lo, Flow localization in the plane strain tensile test, J. Mech. Phys. Solids 29 (1981) 15-142.

[4] T. Belytschko, J. Fish, B. E. Engelmann, A finite element with embedded localization zones, Computer methods in applied mechanics and engineering 70 (1988) 59-89.

[5] D. Bigoni, D. Capuani, Green's function for incremental nonlinear elasticity: shear bands and boundary integral formulation, Journal of the Mechanics and Physics of Solids 50 (2002) 471-500.

[6] M.Brun, D. Bigoni, D. Capuani, A boundary element technique for incremental, non-linear elasticity - Part II: Bifurcation and shear bands, Computer methods in applied mechanics and engineering 192 (2003) 2481-2499.

[7] M. Bonnet, Boundary Integral Equation Methods for Solids and Fluids, John Wiley \& Sons, 1999.

[8] J. Rice, The localization of plastic deformation, in: W. Koiter (Ed.), $14^{\text {th }}$ International Congress on Theoretical and Applied Mechanics, NorthHolland, Amsterdam, 1977, pp. 207-220.

[9] A. Needleman, Material rate dependence and mesh sensitivity in localization problems, Computer methods in applied mechanics and engineering 67 (1988) 69-85.

[10] F. Zhou, J.-F. Molinari, T. Shioya, A rate-dependent cohesive model for simulating dynamic crack propagation in brittle materials, Engineering Fracture Mechanics (2005) 1383-1410.

[11] A. Benallal, C. A. Fudoli, W. S. Venturini, An implicit BEM formulation for gradient plasticity and localization phenomena, International journal for numerical methods in engineering 53 (2002) 1853-1869.

[12] H.-B. Mühlhaus, E. C. Aifantis, A variational principle for gradient plasticity, Int. J. Solids Structures 28 (1991) 845-857.

[13] R. De Borst, H.-B. Mühlhaus, Gradient-dependent plasticity: formulation and algorithm aspects, Internatioan journal for numerical methods in engineering 35 (1992) 521-539. 
[14] A. Benallal, A. Botta, W. S. Venturini, On the description of localization and failure phenomena by the boundary element method, Computer methods in applied mechanics and engineering 195 (2006) 5833-5856.

[15] R. De Borst, Simulation of strain localisation: a reappraisal of the Cosserat continuum, Engineering computations 8 (1991) 317-332.

[16] X.-P. Xu, A. Needleman, Numerical simulations of fast crack growth in brittle solids, Journal of the Mechanics and Physics of Solids 42 (1994) 1397-1434.

[17] G. T. Camacho, M. Ortiz, Computational modelling of impact damage in brittle materials, Int. J. Solids Structures 33 (1996) 2899-2938.

[18] A. Pandolfi, M. Ortiz, An efficient adaptive procedure for threedimensional fragmentation simulations, Engineering with computers 18 (2002) 148-159.

[19] F. Zhou, J.-F. Molinari, Dynamic crack propagation with cohesive elements: a methodology to address mesh dependency, International journal for numerical methods in engineering 59 (2004) 1-24.

[20] A. C. Palmer, J. Rice, The growth of slip surfaces in the progressive failure of over-consolidated clay, in: Proceedings of the Royal Society of London A: Mathematical, Physical and Engineering Sciences, volume 332, The Royal Society, pp. 527-548.

[21] E. Van der Giessen, A. Needleman, Discrete dislocation plasticity: a simple planar model, Modelling and Simulation in Materials Science and Engineering 3 (1995) 689-735.

[22] G. Maier, G. Novati, Z. Chen, Symmetric galerkin boundary element method for quasi-brittle-fracture and frictional contact problems, Computational Mechanics 13 (1993) 74-89.

[23] D. A. Hill, P. A. Kelly, D. N. Dai, A. M. Korsunsky, Solution of Crack Problems: the Distributed Dislocation Technique, Kluwer Academic Publishers, 1996.

[24] S. G. Mogilevskaya, Lost in translation: Crack problems in different languages, International Journal of Solids and Structures 51 (2014) 4492-4503. 
[25] S. L. Crouch, A. M. Starfield, Boundary Element Methods in Solid Mechanics, George Allen and Unwin, 1983.

[26] M. Bonnet, G. Maier, C. Polizzotto, Symmetric galerkin boundary element methods, Applied Mechanics Reviews 51 (1998) 669-704.

[27] Itasca Consulting Group, FLAC Constitutive models, Technical report, Itasca Consulting Group, 2010.

[28] F. Ciardo, B. Lecampion, Effect of dilatancy on the transition from aseismic to seismic slip due to fluid injection in a fault, Journal of Geophysical Research: Solid Earth 124 (2019) 3724-3743.

[29] S. Stupkiewicz, Z. Mróz, Modelling of friction and dilatancy effects at brittle interfaces for monotonic and cyclic loading, Journal of theoretical and applied mechanics 3 (2001).

[30] M. Ortiz, A. Pandolfi, Finite-deformation irreversible cohesive elements for three-dimensional crack-propagation analysis, International journal for numerical methods in engineering 44 (1999) 1267-1282.

[31] L. Snozzi, J.-F. Molinari, A cohesive element model for mixed mode loading with frictional contact capability, International journal for numerical methods in engineering 93 (2013) 510-526.

[32] A. Crawford, J. Curran, Higher-order functional variation displacement discontinuity elements, International Journal of Rock Mechanics and Mining Science \& Geomechanics Abstracts 19 (1982) 143-148.

[33] W. Hackbusch, A Sparse Matrix Arithmetic Based on $\mathcal{H}$-Matrices. Part I: Introduction to $\mathcal{H}$-Matrices., Computing 62 (1999) 89-108.

[34] W. Hackbusch, Hierarchical matrices: algorithm and analysis, volume 49, Springer, 2015.

[35] M. Bebendorf, Hierarchical matrices: A Means to Efficiently Solve Elliptic Boundary Value Problems, Springer, 2008.

[36] S. Chaillat, L. Desiderio, P. Ciarlet, Theory and implementation of h-matrix based iterative and direct solvers for helmholtz and elastodynamic oscillatory kernels, Journal of Computational Physics 351 (2017) 165-186. 
[37] A. Quarteroni, R. Sacco, F. Saleri, Numerical mathematics, Springer, 2000.

[38] M. Benzi, G. H. Golub, J. Liesen, Numerical solution of saddle point problems, Acta Numerica (2005) 1-37.

[39] Z.-H. Cao, Augmentation block preconditioners for saddle point-type matrices with singular $(1,1)$ blocks, Numerical linear algebra with applications 15 (2008) 515-533.

[40] Z.-H. Cao, Constraint Schur complement preconditioners for nonsymmetric saddle point problems, Applied Numerical Mathematics 59 (2009) 151-169.

[41] J.-L. Li, T.-Z. Huang, L. Li, The spectral properties of the preconditioned matrix for nonsymmetric saddle point problems, Journal of Computational and Applied Mathematics 235 (2010) 270-285.

[42] S. Li, S. Ahmed, G. Klimeck, E. Darve, Computing entries of the inverse of a sparse matrix using the FIND algorithm, Journal of Computational Physics 227 (2008) 9408-9427.

[43] A. M. Erisman, W. F. Tinney, On computing certain elements of the inverse of a sparse matrix, Numerical mathematics (1975).

[44] F. Maerten, L. Maerten, M. Cooke, Solving 3D boundary element problems using constrained iterative approach, Computational Geoscience 14 (2010) 551-564.

[45] M. L. Cooke, D. D. Pollard, Bedding-plane slip in initial stages of faultrelated folding, Journal of Structural Geology 19 (1997) 567-581.

[46] G. Kirsch, Die Theorie der Elastizität und die Bedürfnisse der Festigkeitslehre, Springer, 1898.

[47] B. Lecampion, Modeling size effects associated with tensile fracture initiation from a wellbore, International Journal of Rock Mechanics and Mining Science 56 (2012) 67-76.

[48] D. Leguillon, D. Quesada, C. Putot, E. Martin, Prediction of crack initiation at blunt notches and cavities: size effects, Eng. Frac. Mech. 74 (2007) 2420-2436. 
[49] J. W. Rudnicki, J. R. Rice, Conditions for the localization of deformation in pressure-sensitive dilatant materials, J. Mech. Phys. Solids 23 (1975) 371-394.

[50] J. Rice, The localization of plastic deformation, Theoretical and Applied Mechanics (Proceeding of the 14th International Congress on Theoretical and Applied Mechanics, Delft, 1976, ed. W. T. Koiter) 1 (1976) $207-220$.

[51] M. Ortiz, Y. Leroy, A. Needleman, A finite element method for localized failure analysis, Computer methods in applied mechanics and engineering 61 (1987) 189-214.

[52] F. Armero, K. Garikipati, An analysis of strong discontinuities in mutiplicative finite strain plasticity simulation of strain localization in solids, Int. J. Solids Structures 33 (1996) 2863-2885.

[53] K. Terzaghi, Theoretical soil mechanics, John Wiley \& Sons, 1943.

[54] D. I. Garagash, L. N. Germanovich, Nucleation and arrest of dynamic slip on a pressurized fault, Journal of Geophysical Research 117 (2012).

[55] C. G. Hatton, I. G. Main, P. G. Meredith, Non-universal scaling of fracture length and opening displacement, Nature 367 (1994).

[56] R. L. Kranz, Microcracks in rocks: a review, Tectonophysics 100 (1983) 449-480.

[57] B. Lecampion, A. P. Bunger, X. Zhang, Numerical methods for hydraulic fracture propagation: A review of recent trends, Journal of Natural Gas Science and Engineering 49 (2018) 66-83.

[58] M. De Bellis, G. Della Vecchia, M. Ortiz, A. Pandolfi, A linearized porous brittle damage material model with distributed frictionalcohesive faults, Engineering Geology 215 (2016) 10-24.

[59] M. L. De Bellis, G. Della Vecchia, M. Ortiz, A. Pandolfi, A multiscale model of distributed fracture and permeability in solids in all-round compression, Journal of the Mechanics and Physics of Solids 104 (2017) $12-31$. 


\begin{tabular}{|l||l|l|l|}
\hline $\mathcal{I}$ & 0.1 & 1 & 10 \\
\hline$p_{b} / p_{b, \text { strength }}$ & 1.052 & 1.448 & 2.906 \\
\hline$p_{b} / p_{b, \text { strength from }[47]}$ & $\sim 1.1$ & $\sim 1.45$ & $\sim 2.9$ \\
\hline
\end{tabular}

Table 1: Comparison of the normalized crack initiation pressure obtained here and the ones of Lecampion [47] for different Irwin numbers $\mathcal{I}$. 


\begin{tabular}{|c||c|c|c|}
\hline & $\eta=0.1$ & $\eta=0.8$ & $\eta=3$ \\
\hline Compression ratio $c_{r}$ & 0.296 & 0.123 & 0.093 \\
\hline Scaled total CPU time & 0.688 & 0.276 & 0.213 \\
\hline Max. rel. difference on half crack length & $0.510^{-3}$ & $1.510^{-3}$ & $1.210^{-2}$ \\
\hline Max. rel. difference on peak slip & $1.4910^{-6}$ & $1.4810^{-6}$ & $3.9610^{-5}$ \\
\hline
\end{tabular}

Table 2: Scaled total CPU time and the maximum relative difference obtained during the simulation for different values of $\eta$ for the hierarchical approximation. The reference numerical solution corresponds to the $\eta=0$ case (no compression of the elasticity matrix). 